\title{
Role of Fault Branches in Earthquake Rupture Dynamics
}

\section{Citation}

Bhat, Harsha S., Marion Olives, Renata Dmowska, and James R. Rice. 2007. Role of fault branches in earthquake rupture dynamics. Journal of Geophysical Research, 112(B11309).

\section{Published Version}

http://dx.doi.org/10.1029/2007JB005027

\section{Permanent link}

http://nrs.harvard.edu/urn-3:HUL.InstRepos:2643852

\section{Terms of Use}

This article was downloaded from Harvard University's DASH repository, and is made available under the terms and conditions applicable to Other Posted Material, as set forth at http:// nrs.harvard.edu/urn-3:HUL.InstRepos:dash.current.terms-of-use\#LAA

\section{Share Your Story}

The Harvard community has made this article openly available.

Please share how this access benefits you. Submit a story.

Accessibility 


\title{
Role of fault branches in earthquake rupture dynamics
}

\author{
Harsha S. Bhat, ${ }^{1}$ Marion Olives, ${ }^{2}$ Renata Dmowska, ${ }^{1,3}$ and James R. Rice ${ }^{1,3}$ \\ Received 2 March 2007; revised 28 July 2007; accepted 29 August 2007; published 29 November 2007.
}

[1] We analyze earthquake ruptures propagating along a straight "main" fault and encountering a finite-length branch fault. Such intersections are often observed in natural fault systems. The predicted effects of the interaction with the branch that we report can be remarkable; they can strongly perturb the propagation velocity on the main fault and, in some cases, even arrest that propagation. Earlier work (Kame et al., 2003; Bhat et al., 2004) emphasized the role of the fault pre-stress state, branch geometry (i.e., branching angle), and the incoming rupture velocity at the branching junction in determining whether the rupture would follow the branch or continue on the main fault or both, through simulations which did not let a rupture on the branch encounter a barrier or a fault end (called 'infinite' branch cases henceforth). In this study we look at "finite" branch cases, and study the effect also of branch length, with rupture being blocked from propagation beyond the branch end. It is known that sudden stoppage of a dynamic rupture front leads to the propagation of large dynamic stress perturbations in the medium. These have been known to nucleate or terminate ruptures on adjacent fault segments (Harris et al., 1991; Harris and Day, 1993, 1999; Harris et al., 2002; Fliss et al., 2005, among others). We thus anticipate interaction between the rupture on the main fault and the branched one at two stages, when the rupture is propagating on the branch and when it is suddenly blocked at the branch end. We show that in general rupture termination on a compressional branch little affects propagation on the main fault compared to the infinite branch cases. For branches on the extensional side, we show in some cases, that whereas an infinite' branch would have allowed (or stopped) rupture propagation on the main fault, a finite branch stops (or allows) propagation on the main fault. Such results have a dependence on branch length that we document. We also illustrate branch-related complexities in rupture velocity evolution which could be one of the sources of the highfrequency content of strong ground motion record. Complexities in the slip distribution, often associated with a presumed heterogeneous strength distribution along the fault, can also be observed when rupture is terminated on a branch.

Citation: Bhat, H. S., M. Olives, R. Dmowska, and J. R. Rice (2007), Role of fault branches in earthquake rupture dynamics, J. Geophys. Res., 112, B11309, doi:10.1029/2007JB005027.

\section{Introduction}

[2] Large earthquake events are complex processes. These complexities show up in the form of short bursts of high-frequency ground motion, branches and offsets in the rupture path, and asymmetry in the inferred slip pattern, to name a few. The source of seismic complexities is normally thought to be associated with heterogeneity in the stress and/or strength distribution along the fault. The other important question of how an earthquake stops is also often

\footnotetext{
${ }^{1}$ School of Engineering and Applied Sciences, Harvard University, Cambridge, Massachusetts, USA.

${ }^{2}$ Laboratoire de Mcanique, Ecole Polytechnique, Palaiseau, France.

${ }^{3}$ Department of Earth and Planetary Sciences and School of Engineering and Applied Sciences, Harvard University, Cambridge, Massachusetts, USA.

Copyright 2007 by the American Geophysical Union. 0148-0227/07/2007JB005027\$09.00
}

attributed to spatial heterogeneity in the strength/stress distribution along the fault. The aim of this study is to explore another mechanism to explain seismic complexities, namely, a class of geometric complexities in the form of branches.

[3] A fault system has in general geometric complexities, long known to geologists [King and Nabelek, 1985; Sibson, 1985, 1986; King, 1986; Wesnousky, 1988; Knuepfer, 1989; Aydin and Schultz, 1990; Yule and Sieh, 2003; Brankman and Aydin, 2004; Wesnousky, 2006, among others], like bends, branches, step-overs, and sub-parallel strands at different length scales (e.g., 1992 Landers earthquake, Figure 1). The interaction between these geometric complexities and rupture has been observed for various earthquakes. For example, in the case of branches, the 1992 Landers event branched from the Johnson Valley Fault to the Kickapoo fault with part of the rupture continuing on the Johnson Valley fault for four kilometers beyond the branch- 


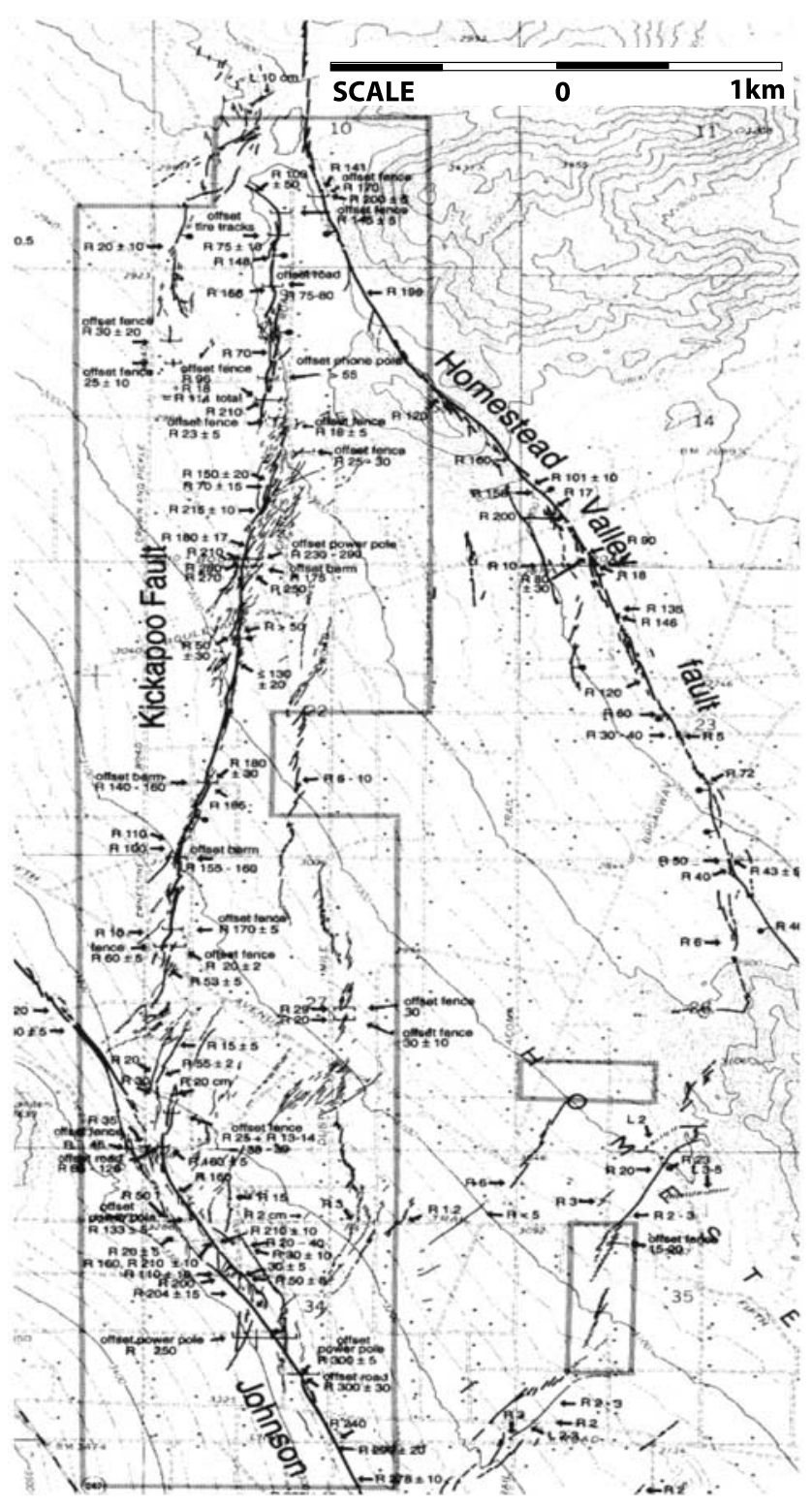

Figure 1. Map from Sowers et al. [1994] showing region of transition from the Johnson Valley to the Kickapoo and to the Homestead Valley faults during the 1992 Landers earthquake. The thickest lines show fault breaks with surface slip $>1 \mathrm{~m}$, intermediate lines $>0.05 \mathrm{~m}$, and thinnest lines $>0.01 \mathrm{~m}$.

ing junction [Sowers et al., 1994; Kame et al., 2003]. The 2002 Denali event branched off from the main Denali fault to the Totschunda fault with no observable surface slip on Denali fault beyond the branching junction [Bhat et al., 2004]. Similar examples of branching from various other earthquakes are discussed by Kame et al. [2003].

[4] Numerical modeling of geometric complexities and their interaction with the rupture process has been done by Tada and Yamashita [1997], Kame et al. [2003] and Bhat et al. [2004], among others, for a rupture branching through a fault system in 2D using the Boundary Integral Equation method, and by Duan and Oglesby [2005] using the Finite Element method. Aochi et al. [2000a, 2000b,
2002, 2005] and Aochi and Fukuyama [2002] addressed similar problems in 3D using the BIE method, and Oglesby et al. [2003], Dreger et al. [2004] and Oglesby [2005] using the FE method.

[5] In this work we emphasize the role of small, finite branches off the main fault in explaining certain complexities associated with the whole rupture process like large scale asymmetry in the slip pattern and even arrest of rupture propagation on the main fault due to the branch.

[6] The paper is organized as following: In section 2 we briefly introduce the numerical methodology and the failure criteria used in our studies. In section 3 we discuss the different parameters used in our model. Section 4 involves discussion of our results. In section 4.1 we compare the differences between effects of infinite and finite branches on propagation along the main fault. In section 4.2 we describe the heterogeneities in stress, slip and rupture velocity observed while varying the parameters. Finally in section 5 we summarize and conclude our studies.

\section{Dynamic Rupture Model}

\subsection{Model Geometry and Properties}

[7] We consider a two dimensional mode II rupture propagating in a medium that is unbounded, homogeneous, isotropic and linear elastic. The rupture propagates rightlaterally as shown by Kame et al. [2003]. We also assume that the slip along the fault is purely tangential and hence do not allow any opening.

[8] The aim is to understand the influence of a finite branch fault [Figure 2] on rupture propagation along a main fault. We compare results with those established by Kame et al. [2003] and Bhat et al. [2004] who analyzed an 'infinite' branch, in the sense that their branch fault had no end, or other imposed barrier-like feature, that was encountered during the time of simulation and hence that would stop the rupture along it.

[9] As proposed by Poliakov et al. [2002], and verified by detailed simulations and refined by Kame et al. [2003] and Bhat et al. [2004], the three key parameters that determine rupture path selection at an infinite branch junction are:

[10] 1. Inclination of the maximum principal compressive stress with the fault, $\Psi$

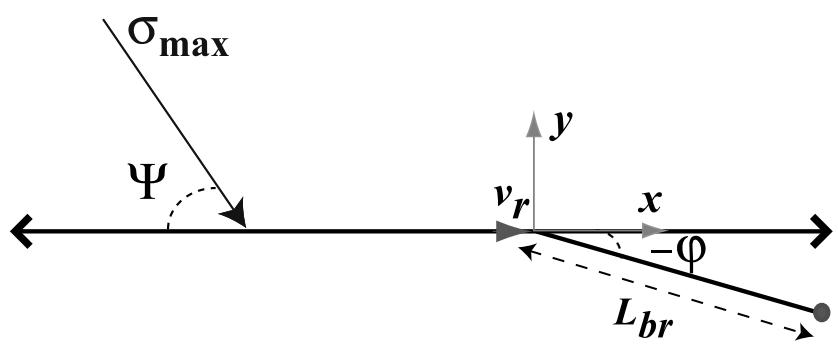

Figure 2. Model geometry and parameters used in the numerical simulations. Here $\Psi$ is the orientation of the maximum principal compressive stress with the main fault, $v_{r}$ is the incoming rupture velocity at the branching junction, $\varphi$ is the inclination of the branch with the main fault and $L_{b r}$ is the length of the finite branch fault. 


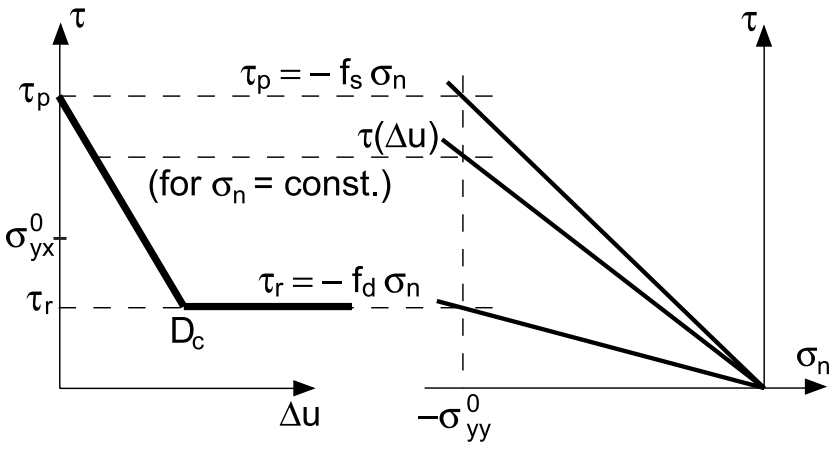

Figure 3. Slip-weakening Coulomb friction law [Kame et al., 2003]. The peak and residual strength $\left(\tau_{p}, \tau_{r}\right)$, and strength $(\tau)$ at any particular amount of slip $(\Delta u)$, is proportional to normal compressive stress $\left(-\sigma_{n}\right)$.

[11] 2. Rupture velocity when approaching a branching junction, $v_{r}$

[12] 3. Fault geometry, the inclination of the branch fault with the main fault, $\varphi$

[13] Kame et al. [2003] have shown that types of faults most susceptible to branch activation are those for which the maximum principal compressive pre-stress direction of the regional stress field is at a steep or shallow angle $\Psi$ relative to what might be regarded as an optimal Coulomb direction, namely $\Psi=45^{\circ}-\phi_{\text {Coulomb }} / 2 \approx 29.5^{\circ}$ for $\tan \left(\phi_{\text {Coulomb }}\right)=$ $\mu_{s}=0.6$. It was shown in their simulations that when $\Psi=25^{\circ}$, the rupture always continued on the main fault and rupture on a branch, if it began at all, soon arrested on it. Thus branch activation is principally a feature of faults which are not near to Coulomb-optimal orientations in their regional pre-stress fields.

[14] Some aspects of the results may also be controlled by the magnitude of the shear prestress, e.g., as measured by the seismic $S$ ratio [Andrews, 1976]. In addition to these parameters, we must now, for finite branches, also introduce the new parameter, $L_{b r}$, which describes the length of the branch. We non-dimensionalize this length by the size of the slip weakening zone at low rupture speeds, $R_{0}$. Rice et al. [2005] have estimated the value of $R_{0}$ to be in the range $1-70 \mathrm{~m}$, with typical sizes of $10-30 \mathrm{~m}$, at mid-seismogenic depth, from their slip pulse model, assuming a peak friction coefficient of 0.6 for a fault with high peak strength and low dynamic strength, and fitting the model to seismic slip inversions by Heaton [1990].

\subsection{Slip-Weakening Coulomb Friction Law}

[15] To describe the failure criterion on the faults, we use the slip-weakening friction law [Ida, 1972; Palmer and Rice, 1973; Andrews, 1976]. As shown in Figure 3, for the simple linear-weakening version, when the normal stress is constant, the shear strength, $\tau$, decreases linearly in this model with ongoing fault slip, $\Delta u$, from a peak strength, $\tau_{p}$ to a residual strength, $\tau_{r}$, and then remains constant after the slip has reached a critical value $D_{c}$.

$$
\tau=\tau_{r}+\left(\tau_{p}-\tau_{r}\right)\left(1-\Delta u / D_{c}\right) H\left(1-\Delta u / D_{c}\right)
$$

where $H($.$) is the Heaviside function.$
[16] We add to this model the Coulomb friction law that describes the shear stress as a linear function of the instantaneous normal stress, $\tau=-f \sigma_{n}$. The friction coefficient, $f$, decreases from an initial value, $f_{s}$, the static friction coefficient, to $f_{d}$, the dynamic friction coefficient, with ongoing fault slip and then remains constant. Thus $\tau_{p}=$ $-f_{s} \sigma_{n}$ and $\tau_{r}=-f_{d} \sigma_{n}$ in equation (1).

[17] This model (slip-weakening Coulomb friction law) has been widely used as a failure criterion in describing earthquake rupture processes because of its simplicity and its ease to implement in numerical methodology. In spite of its simplicity the slip-weakening Coulomb friction law allows the explanation of many complex dynamic rupture processes.

\subsection{Rupture Nucleation}

[18] There are different ways of nucleating a rupture. We could have imposed a very high initial shear stress along the nucleation zone, greater than the failure stress, so that the crack would have been statically unstable or imposed a reduction of normal stress there. The other way of nucleating rupture is to impose a slip distribution, compatible with the slip weakening law along the total crack length equal to the nucleation size, $L_{\text {nucl }}$, and zero everywhere else. This will produce stress concentration, higher than the failure criterion, near the crack tips if $L_{n u c l}>L_{c}$. This minimal nucleation size (which refers here to the total crack length), $L_{c}$, is derived from the fracture mechanics energy balance and has a simple expression when using the slip-weakening Coulomb friction law, if we assume as done by Palmer and Rice [1973] and Rice [1968] that the slip-weakening zone size, $R_{0}$, for low rupture velocities is small compared to all geometric dimensions of the model. Further if, as done by Palmer and Rice [1973], we consider that in the slipweakening zone the shear stress varies linearly with distance along the crack within the end zone, then with a Poisson ratio equal to $0.25(\lambda=\mu)$, we get

$$
L_{c}=\frac{16 \mu G}{3 \pi\left(\sigma_{y x}^{o}-\tau_{r}\right)} ; \quad R_{0}=\frac{9 \mu G}{4 \pi\left(\tau_{p}-\tau_{r}\right)^{2}}
$$

where $G=\int_{o}^{\infty}\left(\tau(\Delta u)-\tau_{r}\right) d(\Delta u)$ is the fracture energy for the slip weakening model, $\mu$ is the shear modulus of the medium and $\sigma_{y x}^{o}$ is the initial shear stress in the medium.

[19] The size of the slip-weakening zone, $R$, depends on the rupture velocity [Rice, 1980; Poliakov et al., 2002; Rice et al., 2005; Bhat et al., 2007], at least for essentially steady state dynamic rupture configurations with $v_{r}$ nearly uniform over a transit time $R / v_{r}$. In the range $v_{r}<$ Rayleigh wave speed $c_{R}$ of the medium, of primary interest here, it diminishes with increasing rupture velocity to reach the value of zero at the limiting speed $c_{R}$, as

$$
R=\frac{R_{0}}{F\left(v_{r}\right)}
$$

where $F\left(v_{r}\right)$ increases from one to infinity when $v_{r}$ increases from 0 to $c_{R}$. To obtain proper results, the slip-weakening zone should be adequately resolved in any numerical model. As shown by Kame et al. [2003] we take the cell size, $\Delta s$, equal to $R_{0} / 5$ for low and intermediate rupture 


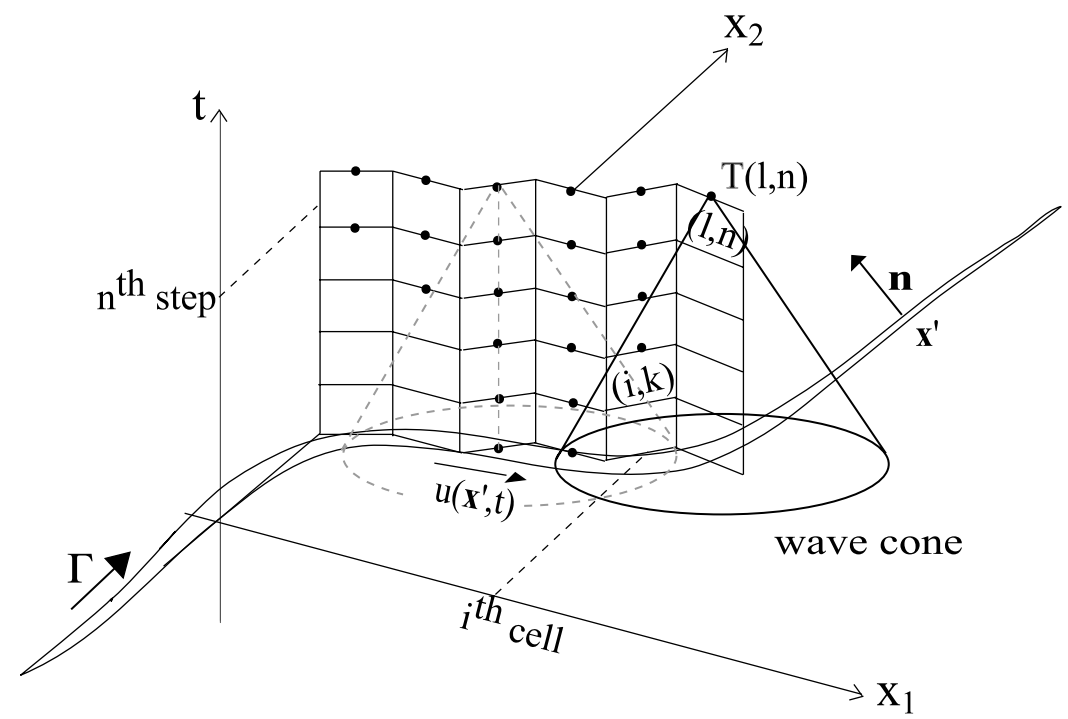

Figure 4. Nomenclature used and schematic diagram of the discretized BIE method. The points represent the cells with non-zero slip velocity [Fliss et al., 2005].

velocities ( $v_{r}=0.60 c_{s}$ and $0.80 c_{s}$ respectively) and $\Delta s=$ $R_{0} / 10$, and for the high velocity $v_{r}=0.90 c_{s}$.

\subsection{Rupture Propagation}

[20] Assuming that the normal stress is compressive $\left(\sigma_{n}<0\right)$, the rupture will propagate the following way. If $\tau>-f_{d} \sigma_{n}$ the rupture will slip right-laterally; else, if $\tau<f_{d} \sigma_{n}$ the rupture will slip left-laterally. Here, we focus on right-lateral rupture. If the slip-velocity is predicted to be negative we then set it to zero. We do not allow any backward slipping, i.e., we do not allow left-lateral slip.

\subsection{Boundary Integral Equation (BIE) Method}

[21] Boundary integral equation methods are very efficient for the study of cracks in homogeneous media, in that the calculations are done only along the crack trace which therefore allows the study of complex crack geometries.

[22] This method derives from the basic integral representation theorem in dynamic elasticity, [e.g., Aki and Richards, 1980]. T. Tada et. al, Boundary Integral Equations for the Time-Domain and Time-Independent Analyses of 2D Non-Planar Cracks, submitted to Earth and Planetary Physics, 1995, hereinafter referred to as T. Tada et. al, submitted manuscript, 1995 has calculated the change in tangential and normal stresses due to a mode II rupture along an arbitrarily shaped crack. A discretized version of those stresses were calculated in earlier works by Koller et al. [1992], Cochard and Madariaga [1994], Kame and Yamashita [1999a, 1999b; Kame et al., 2003], Kame et al. [2003], and Fliss et al. [2005] using piecewise constant interpolation cells. The resulting stresses are, assuming that the discretized slip velocity $V_{i}^{k}$ is constant within each spatial element and during each time step $k$ [Figure 4].

$$
\begin{gathered}
\Delta \tau_{l}^{n}=K_{o}^{o} V_{l}^{n}+\sum_{k=1}^{n-1} \sum_{i} K_{\text {tangential }}^{l, i, n-k} V_{i}^{k}+\tau_{l}^{o} \\
\Delta \sigma_{l}^{n}=\sum_{k=1}^{n-1} \sum_{i} K_{\text {normal }}^{l, i, n-k} V_{i}^{k}+\sigma_{l}^{o}
\end{gathered}
$$

[23] In these expressions $k, n$ are the discretized time indices, and $i, l$ the discretized spatial indices. $\tau_{l}^{o}$ and $\sigma_{l}^{o}$ are respectively the 'pre' shear and normal stresses. $\Delta \tau_{l}^{n}$ and $\Delta \sigma_{l}^{n}$ are respectively the changes in shear an normal stresses at time step $n . K_{o}^{o}\left(=-\mu / 2 c_{s}\right)$ is the radiation damping term [Cochard and Madariaga, 1994; Rice, 1993]. It represents the instantaneous contribution of the current slip-velocity at a certain point to the shear stress at the same point. An instantaneous term does not appear in the expression of the normal stress as long as we consider no opening of the crack. $K^{l, i, n-k}$ is the dynamic stress kernel which represents the influence of unit slip-velocity in cell $i$ at time step $k$ on cell $l$ at time step $n$. These equations show the contribution of the whole fault stress history (within the causality cone) to the shear stress at one point of the fault.

\section{Choice of Parameters}

[24] Different parameters play a major role when explaining the propagation of an earthquake, such as the state of stress in the region before the earthquake nucleates, the rupture velocity, the direction of propagation of the fault and the length of the fault [Poliakov et al., 2002; Kame et al., 2003].

\subsection{Influence of Rupture Velocity}

[25] Some earthquakes are observed to be very slow and others propagate very quickly. In their two dimensional steady state slip pulse model, Rice et al. [2005], building on the semi-infinite mode II crack model of Poliakov et al. [2002], have shown that the closer is the rupture velocity $v_{r}$ to the Rayleigh wave speed of the medium, the larger is the off-fault stress concentration. This stress concentration is mainly around the rupture tip and extends away from the fault to distances of the order of the size of the slip weakening zone in the low rupture velocity, low stress drop limit, as shown in Figure 5.

[26] Thus different rupture velocities, when approaching the branching point, may or may not nucleate rupture on the 


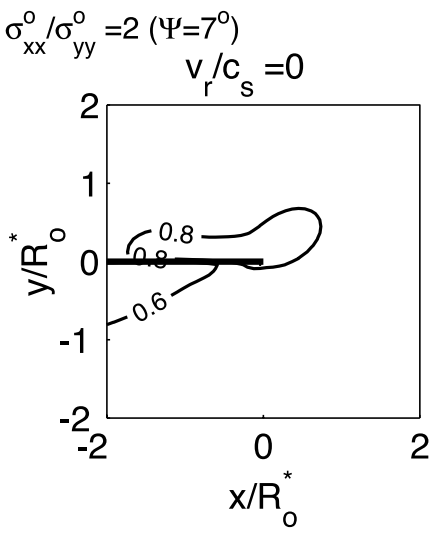

$\sigma_{\mathrm{xx}}^{0} / \sigma_{\mathrm{yy}}^{\circ}=0.8\left(\Psi=64^{\circ}\right)$

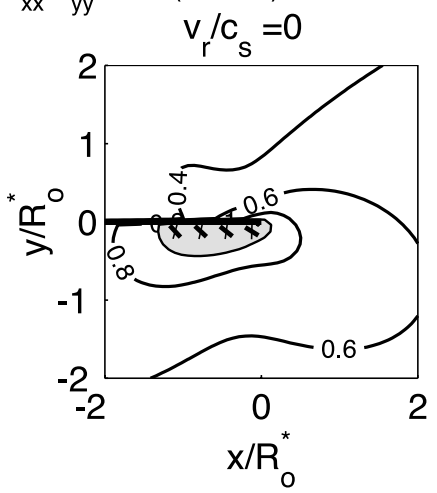

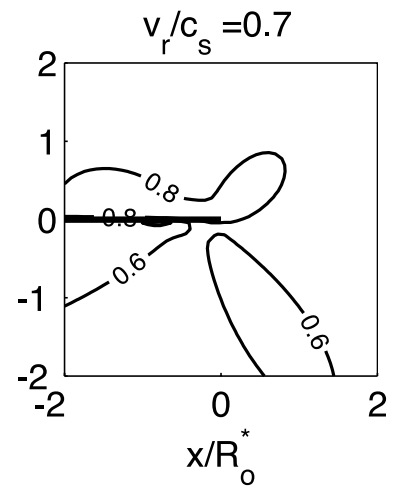

(a)

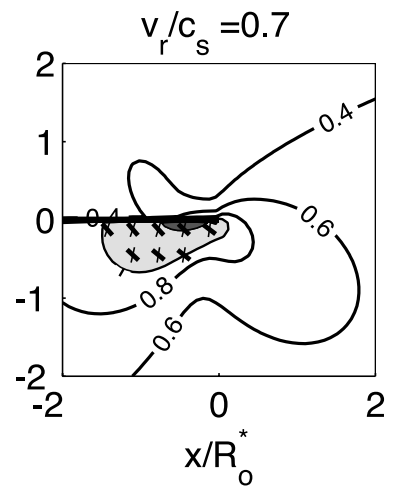

(b)
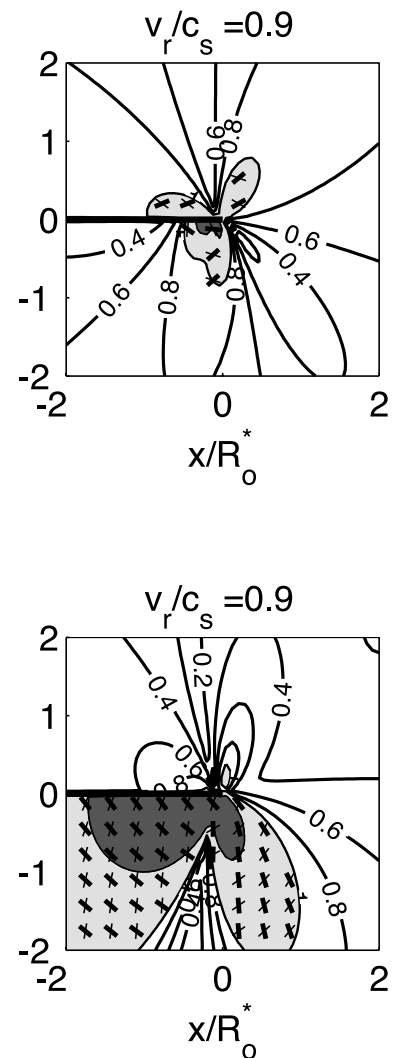

Figure 5. (a) Elastically predicted regions of cohesionless Mohr-Coulomb failure around a slip pulse propagating with a steady speed of $v_{r}$, scaled by the shear wave speed, $c_{s}$. Fault constitutive behavior characterized by spatially linear strength weakening criterion with the peak strength, $\tau_{p}=-0.6 \sigma_{y y}^{o}$ and the residual strength, $\tau_{r}=0.2 \tau_{p}$ at pre-stress ratio $\sigma_{x x}^{o} / \sigma_{y y}^{o}=2.0$. The size of the slip weakening zone, $R$, is 0.001 times the length of the slip pulse, $L . R_{0}^{*}$ is the value of $R$ in the low rupture velocity, low stress drop, limit. The light gray region represents the zone of potential Mohr-Coulomb failure and the failure planes are shown. The dark line represents the main fault. The dark gray region represents the region where one of the principal stress components turns tensile. (b) Same as (a) except $\sigma_{x x}^{o} / \sigma_{y y}^{o}=0.8$. [Rice et al., 2005 ].

branched fault. We choose three values for the rupture velocity near the branching region, $0.60 c_{s}, 0.80 c_{s}$, and $0.90 c_{s}$, low, medium and high rupture velocity respectively, characterized by Kame et al. [2003]. We control the rupture velocity, when approaching the branching junction, by nucleating rupture at various distances from the branching junction as shown in Figure 6.

\subsection{Friction Coefficient and Fracture Energy}

[27] The slip-weakening law depends on the values of $f_{d}$ and $f_{s}$, the friction coefficients, and on $D_{c}$, the critical slip. Poliakov et al. [2002] and Rice et al. [2005] showed that the ratio $f_{d} / f_{s}$ has a large influence on the shape of the highstress region. For our study we take the same values as those by Kame et al. [2003], which are $f_{s}=0.60$ (typical value for crustal rocks) and $f_{d}=0.12$ (chosen arbitrarily).

\subsection{Influence of Initial Stress}

[28] From Poliakov et al. [2002] and Kame et al. [2003] we know that although rupture velocity plays a key role in triggering high off-fault stresses, the sustenance of rupture on a branch is controlled by the inclination of the principal maximum compressive stress with respect to the main fault. All stresses can be normalized by the initial compressive normal stress, $-\sigma_{y y}^{o}$ ( $\sigma_{y y}^{o}$ is positive in tension), on the main fault. The normalized shear stress was fixed at 0.24 to set the seismic $S$ ratio, $\left(\tau_{p}-\sigma_{x y}^{o}\right) /\left(\sigma_{x y}^{o}-\tau_{r}\right)$, Andrews [1976], to be equal to 3 (which leads to sub-Rayleigh rupture). $\sigma_{x x}^{o} / \sigma_{y y}^{o}$ was then chosen to fix the inclination of the principal maximum compressive stress on the main fault.

[29] Poliakov et al. [2002] concluded that generally for low principal maximum compressive stress inclination $\Psi$ with respect to the main fault, the high stress region (in the sense of proximity to Mohr-Coulomb failure conditions) would be on both sides of the fault as in Figure 5a. For large values of $\Psi$, especially angles greater than $45^{\circ}$, it would be mainly on the extensional side (Figure $5 \mathrm{~b}$ ).

[30] Kame et al. [2003] and Bhat et al. [2004] showed in their numerical simulations that with increasing orientation angle $\Psi$ of the maximum principal compressive stress with respect to the main fault, the favored branch for rupture propagation changes from the one on the compressional side to the one on extensional side. Also, the rupture path becomes more and more exclusively along the branch when 

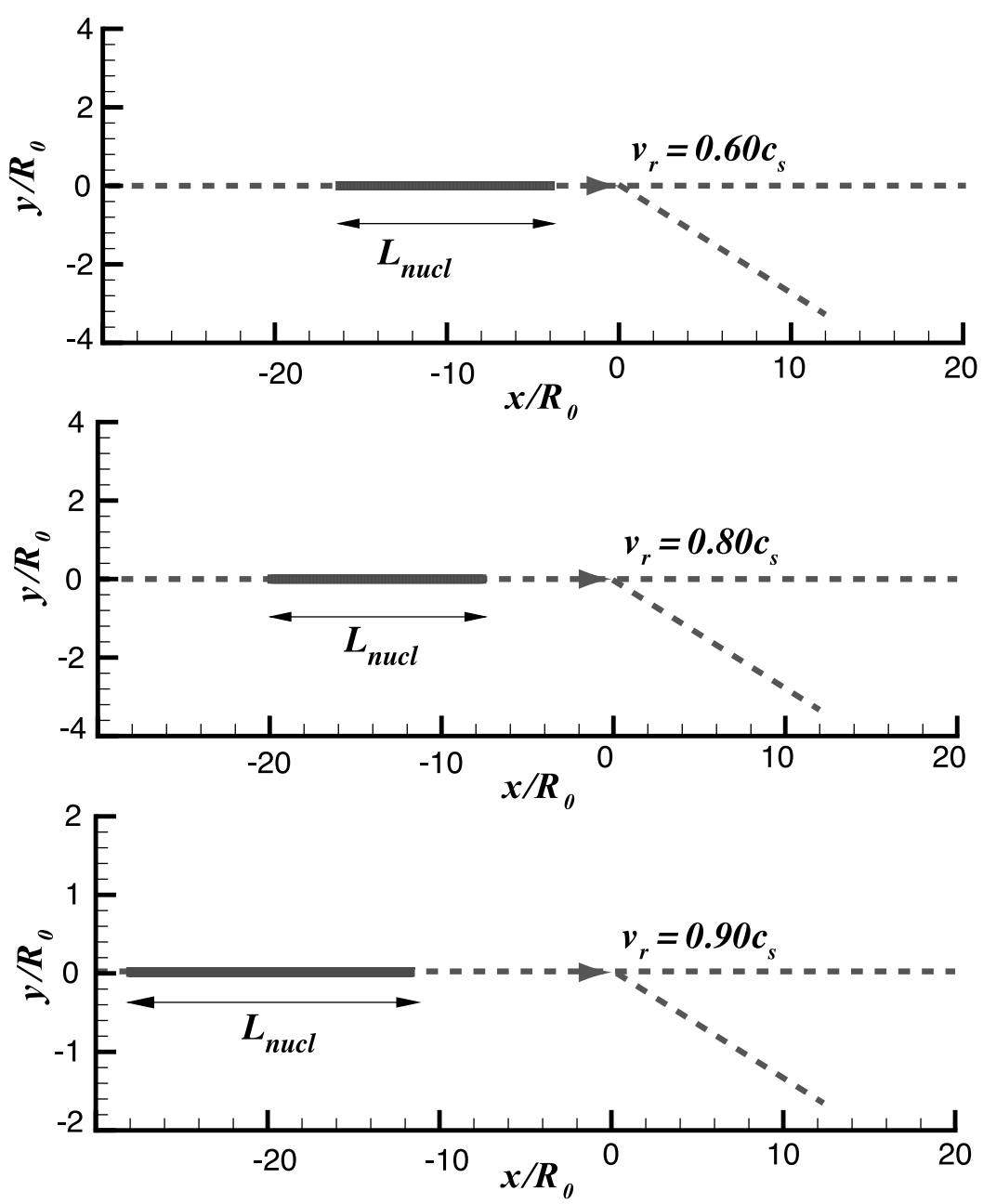

Figure 6. Position of the branch with respect to the nucleation zone in order to achieve different rupture velocities at the branching junction.

the extreme values $\left(0^{\circ}\right.$, for a compressional right-laterally slipping branch, and $90^{\circ}$, for an extensional right-laterally slipping branch) of the maximum principal compressive stress orientation are approached. This exclusivity is lost with increasing rupture velocity but once again, as the limits of $\Psi$ are reached, the exclusivity becomes more and more independent of rupture velocity.

[31] We choose $\Psi=13^{\circ}, 56^{\circ}$ and $70^{\circ}$ in our simulations to compare our results with those for infinite branches of Kame et al. [2003] and Bhat et al. [2004], for different branch angles and rupture velocities near the branching junction.

\subsection{Influence of the Branch Orientation With Respect to the Main Fault}

[32] Since the extensional and the compressional sides of a fault are stressed asymmetrically the final rupture pattern depends on the orientation of the branch fault with the main fault, $\varphi$, (i.e., with the $x$ axis) as shown by Kame et al. [2003].

[33] We consider different inclinations of the branch, with respect to the main fault, that have been shown to have dynamically ruptured by Kame et al. [2003]. We thus consider for $\Psi$ equal to $13^{\circ}$, equal to $15^{\circ}$ and $30^{\circ}$ (compressional side), and for $\Psi$ equal to $56^{\circ}$ and $70^{\circ}$, equal to $-15^{\circ}$ and $-30^{\circ}$ (extensional side).

\subsection{Influence of the Length of the Branch}

[34] Maps of surface slip of large earthquakes [e.g., Sowers et al., 1994] reveal many minor branches, along and beyond the damage zone of a fault structure, and that these branches are of varying lengths with mapped lengths extending from one hundred to a few hundreds of meters. For example, during the 1992 Landers event, one can hypothesize that the main rupture made several attempts to branch on the extensional side of the Johnson Valley fault before finally branching off to the Kickapoo fault. The influence of these features has not been studied precisely yet. The small ones have always been neglected until now while the larger ones were considered to be infinite, effectively, for purposes of analyzing whether the branch path was followed. In this work we try to explain the role of finite branches, specifically their length, $L_{b r}$, on rupture propagation along the main fault.

\section{Discussion of Results}

\subsection{Role of Finite Branches as Compared to 'Infinite'} Branches

[35] A very detailed study of rupture propagation along a branched fault system had been conducted by Kame et al. [2003] for the case where rupture was never terminated on 
the branch ('infinite' branch case). The three main parameters delineated by them, based on the earlier work by Poliakov et al. [2002], were the orientation angle $\Psi$ of the maximum principal compressive direction of the pre-stress field with respect to the main fault, the incoming rupture velocity $v_{r}$ at the branching junction, and the orientation angle of the branch fault with respect to the main fault. Bhat et al. [2004], making use of the Boundary Integral Equation Method for the same set of parameters mentioned above, tried to explain the exclusivity of the branching phenomenon observed during the 2002 Denali Fault earthquake in Alaska.

[36] We would like to categorize the results of Kame et al. [2003] and Bhat et al. [2004], where branching was observed, into two cases, as follows:

[37] 1. The rupture took the branch exclusively and stopped on the main fault.

[38] 2. The rupture propagated on both the main and the branch fault.

[39] The goal here is then to study the effect of finite short and long branches on the rupture propagation characteristics along the main fault, as compared to the infinite branches of Kame et al. [2003] and Bhat et al. [2004].

[40] Kame et al. [2003] observed that for extreme inclinations of $\Psi\left(=13^{\circ}\right.$ and $\left.56^{\circ}\right)$, the rupture almost always propagated, exclusively, on the compressional $(\varphi>0)$ or the extensional branch $(\varphi<0)$, respectively. This exclusiveness was no longer observed when either the branch angle was high $\left(\varphi=30^{\circ}\right.$ or $\left.-30^{\circ}\right)$ or when the rupture velocity was close to the limiting speed, the Rayleigh wave speed. In both these cases, the authors hypothesized that the stressshadow effect was less dominant (due to high rupture velocity or high inclination of the branch) making the two propagating ruptures, on the main and the branch fault, almost independent of each other. Bhat et al. [2004] observed exclusivity of branching with the 2002 Denali fault earthquake, where $\Psi=70^{\circ}$ with $\varphi=-15^{\circ}$, for low and intermediate values of rupture velocities.

[41] We hence chose the above mentioned values for $\Psi\left(\varphi=13^{\circ}, 56^{\circ}\right.$ and $\left.70^{\circ}\right)$ and considered branch angles for which rupture was taken along the branch in the infinite branch case. These are $\varphi=15^{\circ}$ and $30^{\circ}$ for $\Psi=13^{\circ}$ and $\varphi=$ $-15^{\circ}$ and $-30^{\circ}$ for $\Psi=56^{\circ}$ and $\varphi=-15^{\circ}$ (the only case studied by Bhat et al. [2004]) for $\Psi=70^{\circ}$. We also consider all incoming rupture velocities at the branching region, namely $v_{r}=0.60 c_{s}, 0.80 c_{s}$ and $0.90 c_{s}$ as done by Kame et al. [2003] and Bhat et al. [2004].

\subsection{Case With Exclusive Branching}

[42] When $\Psi=13^{\circ}$ and when the branch was inclined at an angle of $15^{\circ}$ to the main fault, the Kame et al. [2003] infinite branch analysis showed that irrespective of the incoming rupture velocity at the branching junction, the rupture always stopped on the main fault almost immediately beyond the branching junction. There was also stoppage of rupture on the main fault when the branch angle was $30^{\circ}$ but only at the lowest speed studied, $v_{r}=0.60 c_{s}$.

[43] When the rupture on the branch is terminated due to finite branch length, in simulations analogous to those discussed in the previous paragraph, we observe some very interesting dynamical behavior of rupture on the main fault. For the case when $\Psi=13^{\circ}, \varphi=15^{\circ}$, the perturbation of the stress field on the main fault during rupture propagation on the branch was sufficient to terminate propagation on the main fault, irrespective of the incoming rupture speed, for both short $\left(L_{b r}=6 R_{0}\right)$ and long branches $\left(L_{b r}=30 R_{0}\right.$ for $v_{r}=$ $0.60 c_{s}, 0.80 c_{s}$ and $L_{b r}=20 R_{0}$ for $v_{r}=0.90 c_{s}$ ). For the above case, when $v_{r}=0.80 c_{s}$, we studied the precise sensitivity of rupture to branch length and noticed that only when the branch is extremely short, $L_{b r}=1 R_{0}$, rupture continues to propagate on the main fault. This shows that extremely short branches (lengths of the order of $R_{0}$ ) can cause earthquake ruptures to renucleate on the main fault, at least on faults like this one which are in a regional stress field which is poorly aligned relative to the optimal alignment for Coulomb rupture on the main fault $\left(\Psi=13^{\circ}\right.$ vs. $\Psi_{\text {Coulomb }}=29.5^{\circ}$ based on $f_{s}=0.60$ ). These results for the finite branch cases (except for branch lengths of the order of $R_{0}$ ) could have been suspected from the infinite branch case [Figure 7], in which rupture chose to follow the branch and soon abandoned the main fault.

[44] For branches on the extensional side, exclusive branching was observed by Kame et al. [2003] in the infinite branch case when $\Psi=56^{\circ}$ and $\varphi=-15^{\circ}$ when $v_{r}=$ $0.60 c_{s}$ and $v_{r}=0.80 c_{s}$ [Figure 7]. For the above cases, the rupture once again stops on the main fault beyond the branching point for both short $\left(L_{b r}=6 R_{0}\right)$ and long branches $\left(L_{b r}=30 R_{0}\right)$ for $v_{r}=0.60 c_{s}$ and for long branch $\left(L_{b r}=30 R_{0}\right)$ when $v_{r}=0.80 c_{s}$.

[45] When the length of the branch was reduced from $30 R_{0}$ to $10 R_{0}$ and when the incoming rupture velocity was $0.80 c_{s}$, the rupture slowed down and stopped temporarily on the main fault until the large perturbation in the stress field, due to the stoppage of rupture on the branch, reached the main fault to kick-start the rupture again on the main fault. Thus a short enough branch (with a length up to $10 R_{0}$, Figure 8), with an intermediate rupture velocity like $0.80 c_{s}$ on the main fault, was sufficient to renucleate the rupture on the main fault. Figure 9 shows this phenomenon quite clearly. When the branch length was $6 R_{0}$, the scaled shear stress evolution on the main fault around the branching junction shows clear propagation of the rupture front, corresponding to the peaks in the figure. The time steps shown in the plots correspond to the time when the rupture front on the main fault was approaching the branching junction to the time when the rupture has completely stopped on the branch. When the branch length was increased to $30 R_{0}$ we see the peaks in the shear stress never reaching the peak strength $\left(\tau_{p}=0.6 \sigma_{y y}\right)$ resulting in no further rupture propagation.

[46] When $\Psi=70^{\circ}$ and $\varphi=-15^{\circ}$ Bhat et al. [2004] observed in the infinite branch case that the rupture stops on the main fault for low and intermediate rupture velocities $\left(v_{r}=0.60 c_{s}\right.$ and $\left.v_{r}=0.80 c_{s}\right)$ [Figure 7].

[47] For branch lengths greater than $6 R_{0}$ our simulations show that a finite branch has the same effect on the rupture on the main fault, i. e. the rupture terminates on the main fault, although the distance covered by the rupture, before stopping, on the main fault depended on the branch length. We also studied sensitivity to branch length for the particular case when the incoming rupture velocity was $0.80 c_{s}$. We introduced branches of length $1,2,3,4,5$ and $6 R_{0}$ and studied their effect of rupture propagation characteristics on the main fault. We observe a transition length of the branch, at $6 R_{0}$, where the rupture on the main fault transitions from 


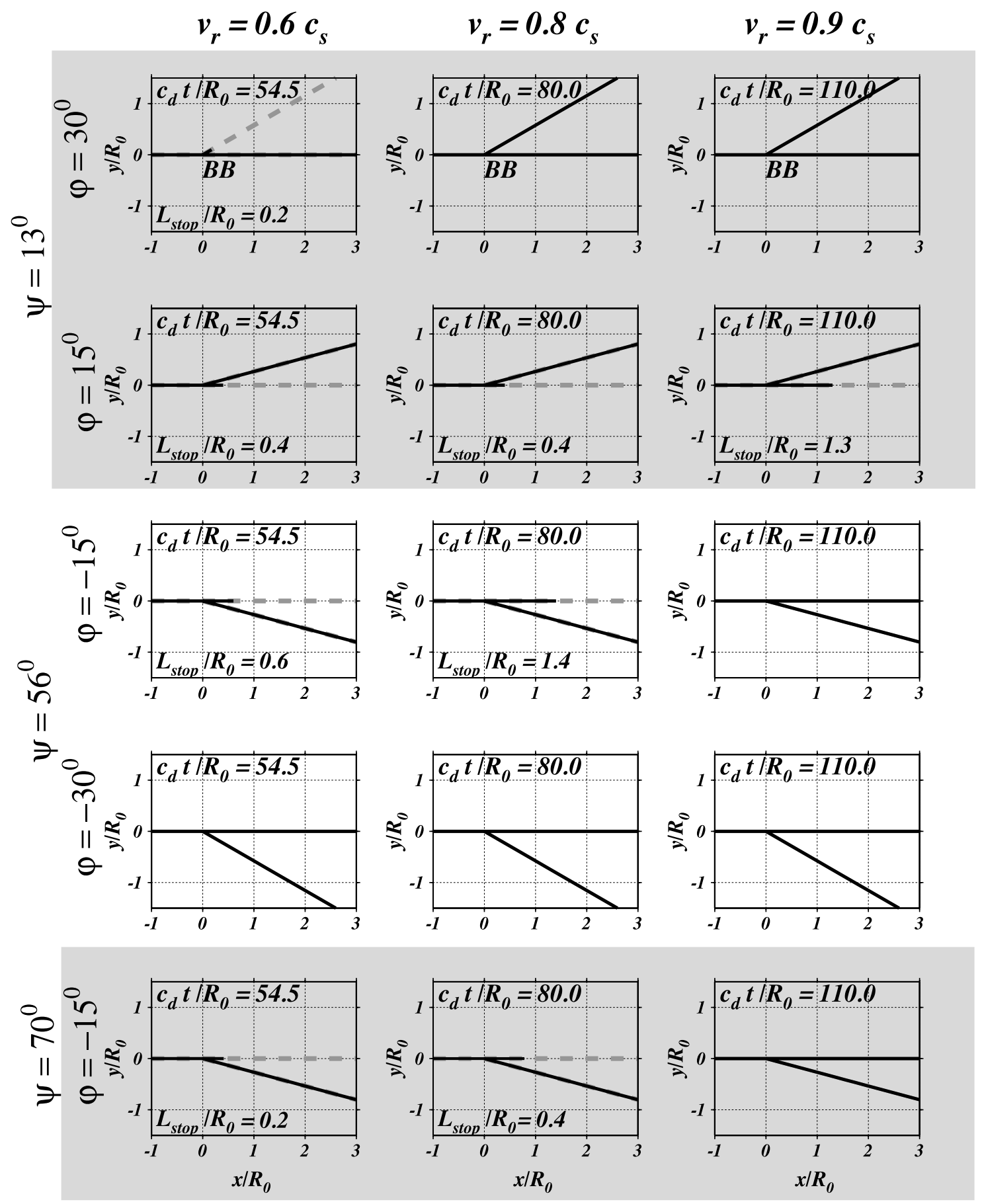

Figure 7. Summary of the results from Kame et al. [2003] and Bhat et al. [2004] "infinite" branch analyses. $L_{\text {stop }}$ indicates the length of stopped rupture front from the branching junction. BB indicates cases with Branching Behind; i.e., the rupture front on the main fault had propagated somewhat beyond the junction before slip initiated on the branch fault. Solid line shows the ruptured part of the fault and the dashed lines represent the unbroken section of the fault.

a continuously propagating state to a state in which it arrests [Figure 10].

[48] Bhat et al. [2004] also studied a case, for the Denali event, in which the approaching rupture velocity at the branching junction was supershear at $v_{r}=1.41 c_{s}$ and they observed exclusive branching. Our simulations of the above case with branch lengths of $6 R_{0}$ and $30 R_{0}$ show that the shorter branch slows the rupture temporarily on the main fault before accelerating it whereas the longer branch halts the rupture on the main fault.

\subsection{Case With Rupture Propagation on Both the Main and the Branch Fault}

[49] For certain orientations of the maximum principal compressive stress with respect to the main fault, branch 


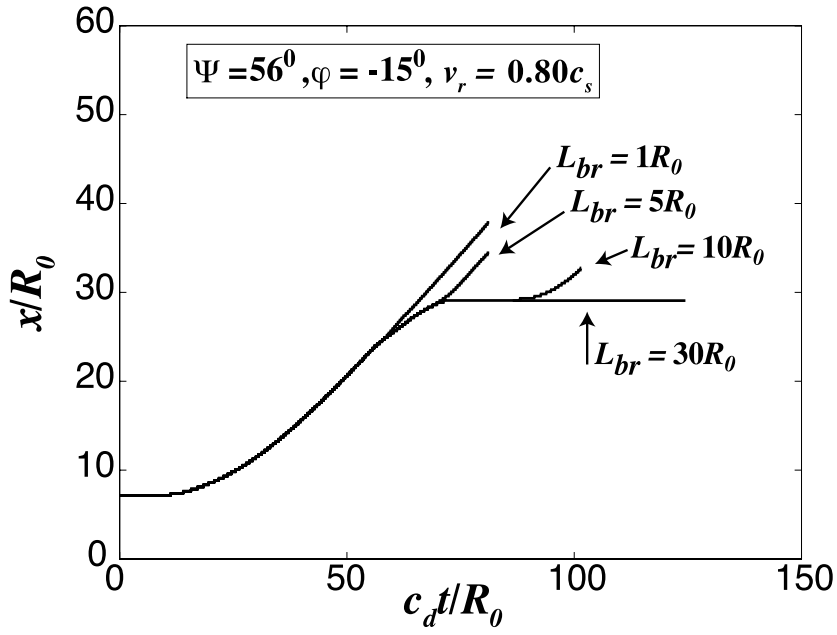

Figure 8. Effect of increasing branch length on the state of rupture front on the main fault (its length is $x$ ), plotted as a function of normalized time, for the case when $v_{r}=0.80 c_{s}$. $L_{b r}$ is the length of the branch, $c_{d}$ and $c_{s}$ are the dilatational and shear wave speeds of the medium respectively.

angles and incoming rupture velocities at the branching junction, Kame et al. [2003] and Bhat et al. [2004] observed, for the infinite branch case, no stoppage of rupture on the main fault once the branch is taken [Figure 7].
[50] For the case when $\Psi=13^{\circ}, \varphi=30^{\circ}$ the termination of rupture on the branch did not alter, from the infinite branch case, in any way the final state of rupture on the main fault for all incoming rupture speeds $\left(v_{r}=0.60 c_{s}, 0.80 c_{s}\right.$, and $\left.0.90 c_{s}\right)$ and branch lengths $\left(L_{b r}=6,30 R_{0}\right.$ for $v_{r}=0.60 c_{s}, L_{b r}=6,8$, $10,20,25 R_{0}$ for $v_{r}=0.80 c_{s}$ and $L_{b r}=6,20 R_{0}$ for $v_{r}=0.90 c_{s}$ ) except for the case when $L_{b r}=30 R_{0}$ and $v_{r}=0.80 c_{s}$. Figure 11 shows the evolution of scaled shear stress on the main fault around the branching junction starting from the time when the rupture is approaching the branching junction to the time when the rupture has completely terminated on the branch for this case. The peaks in the shear stress distribution correspond to the rupture front. When the stress at the peak is less than the shear strength, clearly the rupture front is not able to progress. For $L_{b r}=6 R_{0}$ case we see that for a brief period of time, the rupture front fails to progress before picking up speed again. When $L_{b r}=30 R_{0}$ the magnitude of the peak shear stress decreases with time, even after the rupture has stopped on the branch, indicating the termination of rupture on the main fault. The transition length appears to be between 25 and $30 R_{0}$ [Figure 12].

[51] We suspect this to happen due to the interaction between the rupture front on the main fault and the large stress perturbation emanating from the branch end when rupture stops there. In the short branch case, the rupture on the main fault had already accelerated past the branching junction to a distance where the effect of the branch
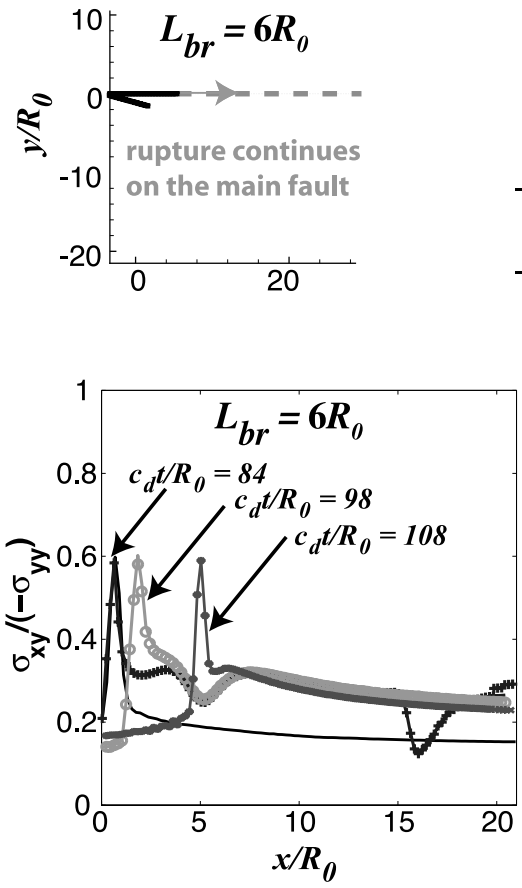

$\Psi=56^{0} ; \varphi=-15^{0} ; v_{r}=0.80 c_{s}$
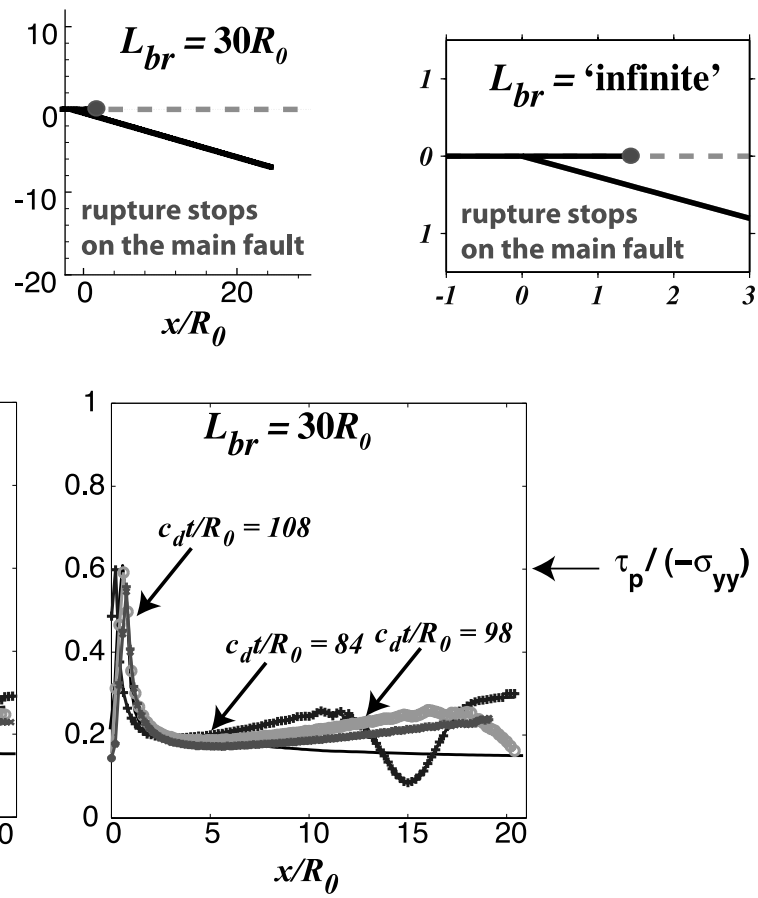

Figure 9. Effect of increasing branch length on the final state of rupture and the corresponding evolution of scaled shear stress distribution on the main fault around the branching junction (stress scaled by fault normal stress, $-\sigma_{y y}$ ) for various time steps (non-dimensionalized as $c_{d} t / R_{0}$ ) for the case when $\Psi=56^{\circ},=-15^{\circ}$ and $v_{r}=0.80 c_{s}$. The time steps correspond to time from when the rupture on the main fault is approaching the branching junction to the time when the rupture has completely stopped on the main fault. $\tau_{p}$ is the peak strength of the fault in the slip-weakening relationship. Here $\tau_{r} / \sigma_{y y}=$ 0.12 and $L_{b r}$ is the length of the branch. 


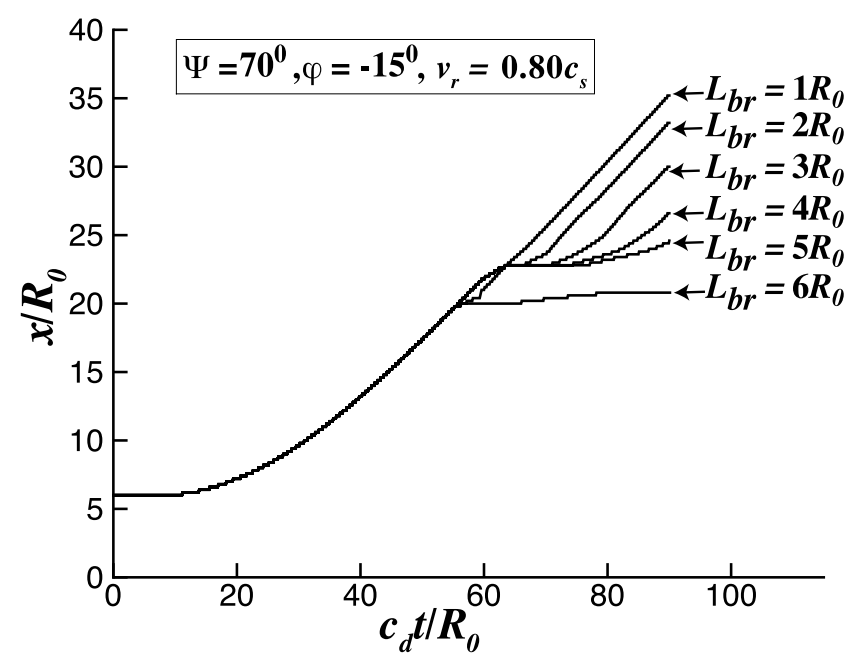

Figure 10. Effect of increasing branch length on the state of rupture front, plotted as a function of normalized time, for the case when $v_{r}=0.80 c_{s} . L_{b r}$ is the length of the branch, $c_{d}$ and $c_{s}$ are the dilatational and shear wave speeds of the medium respectively.

termination is barely felt. Hence the brief slow down and acceleration of the rupture. For long branch length, $L_{b r}>$ $25 R_{0}$, the rupture on the main fault did not accelerate fast enough to outpace the effect of the large stress perturba- tion, emanating due to branch termination, which led ultimately to its stoppage.

[52] When $\Psi=56^{\circ}$ and $\varphi=-15^{\circ}$ and when $v_{r}=0.90 c_{s}$ the rupture propagated both on the main and the branch fault in the Kame et al. [2003] infinite branch simulations. This was attributed by the authors to the reduction in the stress-shadow effect with increasing rupture velocity. Another case where the rupture always continued on the main fault, irrespective of $v_{r}$, after branching was when the branch angle in the above case was increased to $-30^{\circ}$. The relatively high branch angle in this case made the ruptures on the main and the branch fault almost independent of each other irrespective of the incoming rupture velocity at the branch junction [Figure 7].

[53] We notice no significant change in rupture propagation characteristics for the above case when the rupture was terminated on the branch.

[54] Bhat et al. [2004] observed, when trying to numerically simulate the branching phenomenon for the 2002 Denali fault earthquake, that when $\Psi=70^{\circ}$ and $\varphi=-15^{\circ}$ and for extremely high but sub-Rayleigh incoming rupture velocity near the branching junction $\left(0.90 c_{s}\right)$ the rupture propagated on both the main and the branch faults in the infinite branch analysis. [Figure 7].

[55] When the rupture is terminated on the branch then both the short $\left(6 R_{0}\right)$ and long branch $\left(20 R_{0}\right)$ have the effect of stopping the rupture completely on the main fault unlike
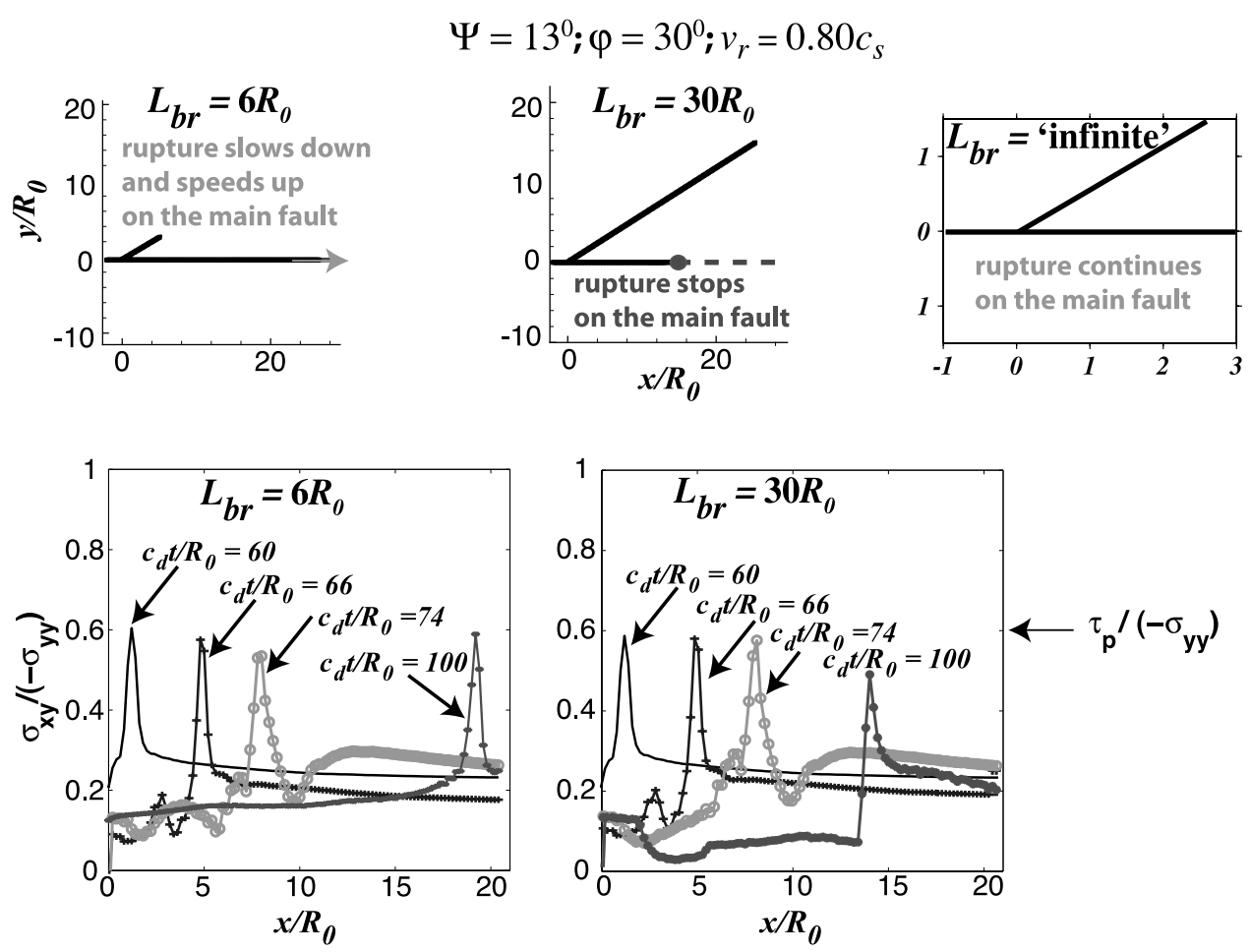

Figure 11. Effect of increasing branch length on the final state of rupture and the corresponding evolution of scaled shear stress distribution on the main fault around the branching junction (stress scaled by fault normal stress, $-\sigma_{y y}$ ) for various time steps (non-dimensionalized as $c_{d} t / R_{0}$ ) for the case when $\Psi=13^{\circ},=30^{\circ}$ and $v_{r}=0.80 c_{s}$. The time steps correspond to time from when the rupture on the main fault is approaching the branching junction to the time when the rupture has completely stopped on the main fault. $\tau_{p}$ is the peak strength of the fault in the slip-weakening relationship. Here $\tau_{r} / \sigma_{y y}=0.12$ and $L_{b r}$ is the length of the branch. In the $L_{b r}=6 R_{0}$ case, the slow down is extreme and involves a brief cessation of rupture propagation on the main fault. 


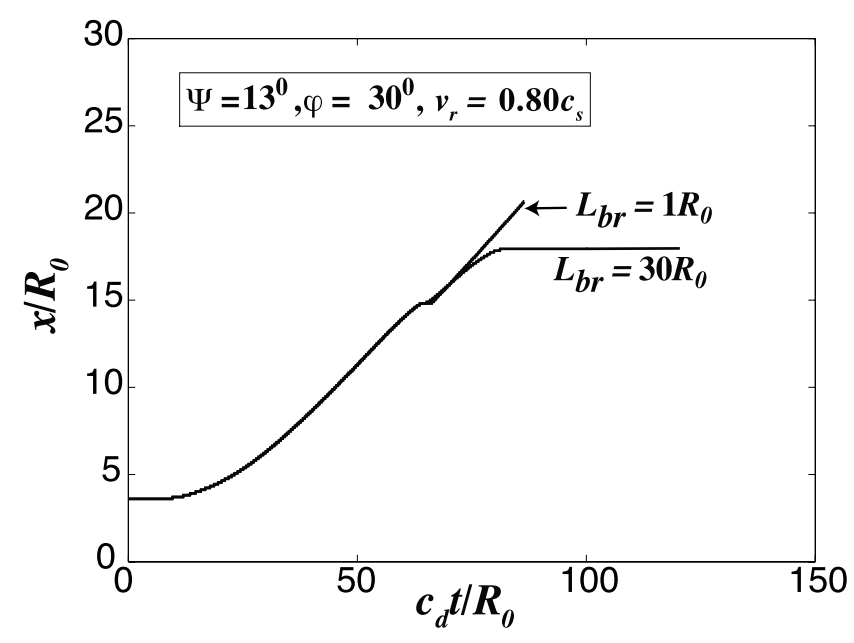

Figure 12. Effect of increasing branch length on the state of rupture front, plotted as a function of normalized time, for the case when $v_{r}=0.80 c_{s}$. $L_{b r}$ is the length of the branch, $c_{d}$ and $c_{s}$ are the dilatational and shear wave speeds of the medium respectively.

the infinite branch case where the rupture propagates on the main fault [Figure 13].

\subsection{Seismic Complexities Due to a Finite Branch}

[56] A dynamically propagating rupture when stopped radiates stress perturbations as it tries to establish a static stress field. The zone of influence of this static-like field depends on many parameters including the length of the rupture. Harris and Day [1993], Harris et al. [2002] and Fliss et al. [2005] have studied the phenomenon of rupture jumping to an adjacent fault due to stoppage on the main fault. In our studies, with finite branched systems, we thus expect some complexity in the rupture propagation process on the main fault due to the stoppage of rupture on the branch.

[57] As outlined in section 4.1, a dynamic shear rupture propagating through a branched fault system behaves quite differently depending on whether the rupture is terminated on the branch or not. It was also noted that the length of the branch plays a key role in influencing the rupture on the main fault. The presence of a finite branch alters the rupture propagation characteristics along the main fault, near the branching junction, as observed in our simulations. In this section we delineate resulting complexities in rupture velocity patterns on the main fault, and on slip patterns, based on the parameters of our model; they are expected to have a bearing on high-frequency ground acceleration.

[58] Two key observations were made regarding the complexities in rupture velocity induced by fault branches. One, there was a general slow down and then a speed up in the rupture velocity and the temporal duration and the spatial extent of this complexity decreased with increasing rupture velocity. Two, there could be a complete slow down and stoppage of rupture propagation on the main fault; that is, the branch could arrest the earthquake.

[59] In the case where the orientation of the maximum principal compressive stress with the main fault, $\Psi$, is $56^{\circ}$ and the inclination of the branch with respect to the main fault, is $-15^{\circ}$ we observe a general slow down and speed up of rupture on the main fault. The duration of this slow down period, before speed up, is longer with increasing length of the branch. When the branch angle is increased to $\varphi=-30^{\circ}$ the effect of the branch in the slowdown-speedup process is also significantly reduced. For the case when $\Psi=13^{\circ}, \varphi=$ $15^{\circ}$ when the length of the branch is $6 R_{0}$ we see complete stoppage of the rupture on the main fault. We in-fact see, for this case, that the rupture stops on the main fault for branch lengths between $2 R_{0}$ and $30 R_{0}$.

[60] The rupture slow down and speed up process decreases both in spatial and temporal extent with increasing rupture velocity near the branching junction. This could be attributed to the fact that as the rupture velocity increases, the stress shadow effect of one fault on the other decreases and the two ruptures behave more and more independently of each other. With increasing rupture velocity near the branching junction two phenomena can be observed.

[61] First, the stress shadow effect of the branch fault on the main fault diminishes. Second, because of the high rupture velocity on the main fault, any factor promoting slow down causes a higher energy flux into the rupture tip, thus mitigating against slow down; that makes it easier for a fast moving rupture to overcome the stress shadow effect of the branch than would its slow moving counterpart, and results in shorter duration of rupture complexities [Figure 14].

[62] Some interesting features can be observed in the slip distribution along the main fault as well. First, on average, the slip deficit on the main fault beyond the branching junction is compensated by the slip on the branch. Secondly, the gradient in slip near the branching junction is higher for

$$
\Psi=70 ; \varphi=-15^{0} ; v_{r}=0.90 c_{s}
$$
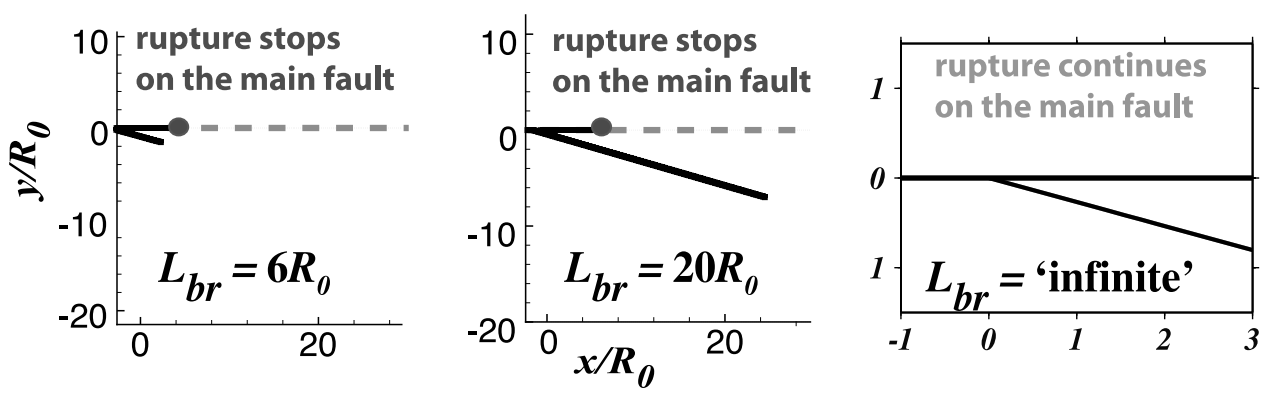

Figure 13. Effect of increasing branch length on the final state of rupture for the case when $\Psi=70^{\circ}$, $\varphi=-15^{\circ}$ and $v_{r}=0.90 c_{s} . L_{b r}$ is the length of the branch. 

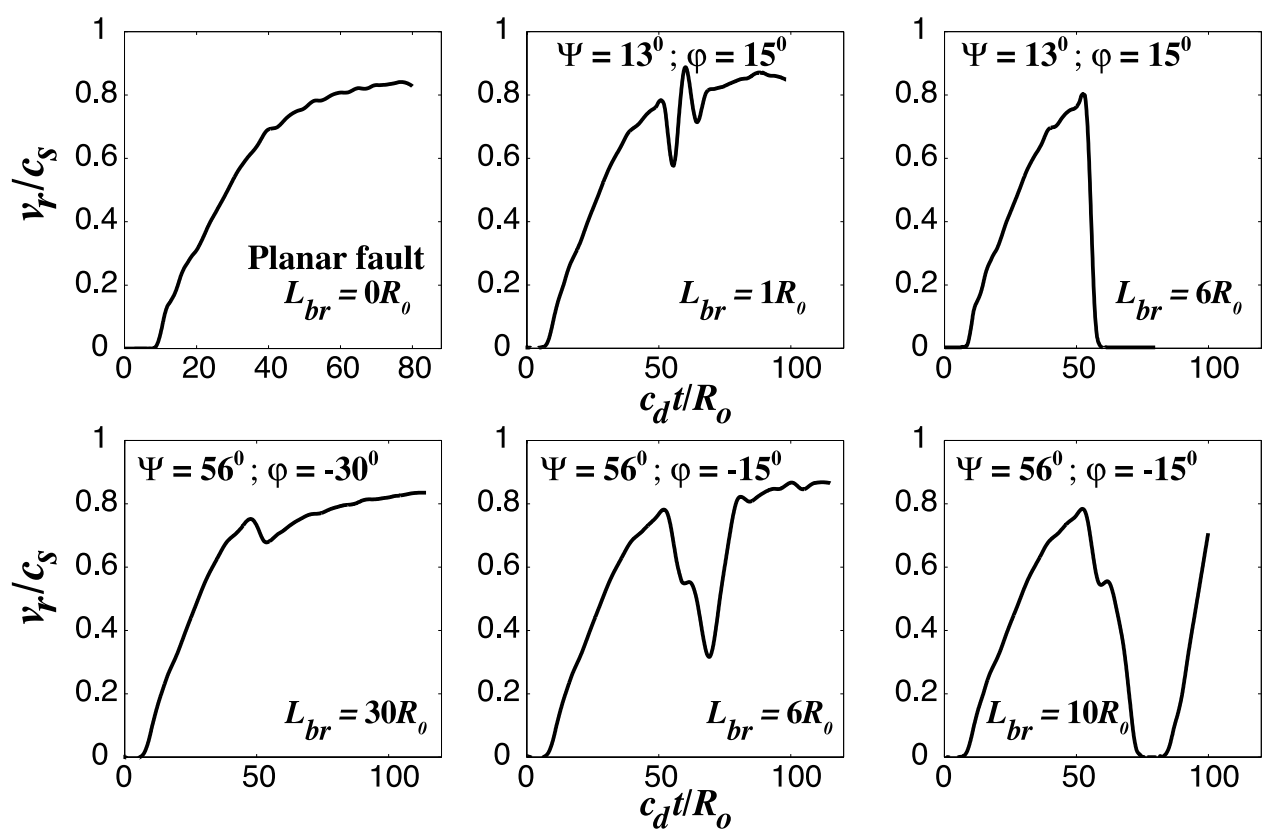

Figure 14. Complexities in the evolution with time of rupture velocity $v_{r}$ (normalized by the shear wave speed $c_{s}$ ) on the main fault, for various configurations. Rupture velocity $v_{r}$ when approaching the branching junction is $0.80 c_{s}$ for all cases.

longer branches than the same for short branches, for cases where finite branches significantly perturbed the rupture process on the main fault. We could attribute this to the strength of the arresting pulse arriving from the end of the branch. From a singular elastic fracture mechanics perspective, the strength of this pulse depends on the rest stress intensity factor, $K_{I I}$, of the branch, which could be expected to increase with $L_{b r}$ and hence be stronger for the long branch than the shorter one. Figures 15 and 16 provide some illustrative slip distribution plots.

[63] In general, sudden gradients in slip distribution on the main fault could be observed when a geometric complexity like a branch is introduced to the main fault. Depending on whether the rupture on the main fault is terminated or not various complexities are introduced in the slip distribution. A sudden drop in the slip accumulation around the branching junction is indicative of rupture termination on the main fault. Small gradients in the slip accumulation process indicate possible slow down and speed up of rupture propagation on the main fault. Figure 17 shows a summary slip distribution on the main fault (when the rupture has terminated on the branch and a static-like stress field is established around it) for all values of $\Psi$, considered when $v_{r}=0.80 c_{s}$ and $L_{b r}=6 R_{0}$.

[64] In some of the above cases (for $\Psi=56^{\circ}$ and $70^{\circ}, v_{r}=$ $0.80 c_{s}$ and $L_{b r}=6 R_{0}$ ) when the branch angle was increased to $-30^{\circ}$ the slow down and speed up process on the main fault is remarkably reduced compared with the same for a smaller branching angle of $-15^{\circ}$ [e.g., see Figure 14]. Also, interestingly, the length of the branch does not then seem to change remarkably the rupture slow down and speed up process on the main fault. This could be because the increased branch angle has already decreased the interaction between the main and the branch fault to such an extent that the length of the branch is then immaterial.
[65] For a branch on the compressional side, $\varphi=15^{\circ}$, and low pre-stress inclination, $\Psi=13^{\circ}$, the slow down and speed up process seems to be more gradual than the same for branches on the extensional side. Of course, the rupture on the main fault slows down for a shorter period of time with increasing rupture velocity. When the branch angle is increased to $30^{\circ}$ the behavior of rupture on the main fault is very interesting. When $v_{r}=0.60 c_{s}$ the slow down in rupture velocity is almost negligible as is the case when $v_{r}=0.90 c_{s}$. However, when $v_{r}=0.80 c_{s}$ there is short period where the rupture slows down before picking up when the branch length is $6 R_{0}$. The same is observed when the branch length is increased to $30 R_{0}$. There is a similar slow down and speed up in rupture velocity before the rupture completely stops on the main fault at a distance of $13 R_{0}$ from the branching junction.

\section{Summary and Conclusions}

[66] We have studied the effect of fault branches on dynamic rupture propagation characteristics along a main fault. This adds to the work of Kame et al. [2003] and Bhat et al. [2004] which focused on the role of pre-stress state, rupture velocity at the branching junction, and the branch angle in controlling rupture path selection on branched fault systems, without terminating the rupture on the branch (referred to as "infinite" branch cases in this study). In brief, their results showed that a shallow branching angle $\left(\varphi= \pm 15^{\circ}\right)$ and a pre-stress state conducive for branching $\left(\Psi=13^{\circ}\right.$ for $\varphi=15^{\circ}$ and $\Psi=56^{\circ}, 70^{\circ}$ for $\left.\varphi=-15^{\circ}\right)$ resulted in rupture termination on the main fault for all rupture velocities, when approaching the branch junction, except high valued ones $\left(v_{r}=0.90 c_{s}\right)$ because of reduced interaction between the main and the branched faults. However, when the orientation of the principal maximum 

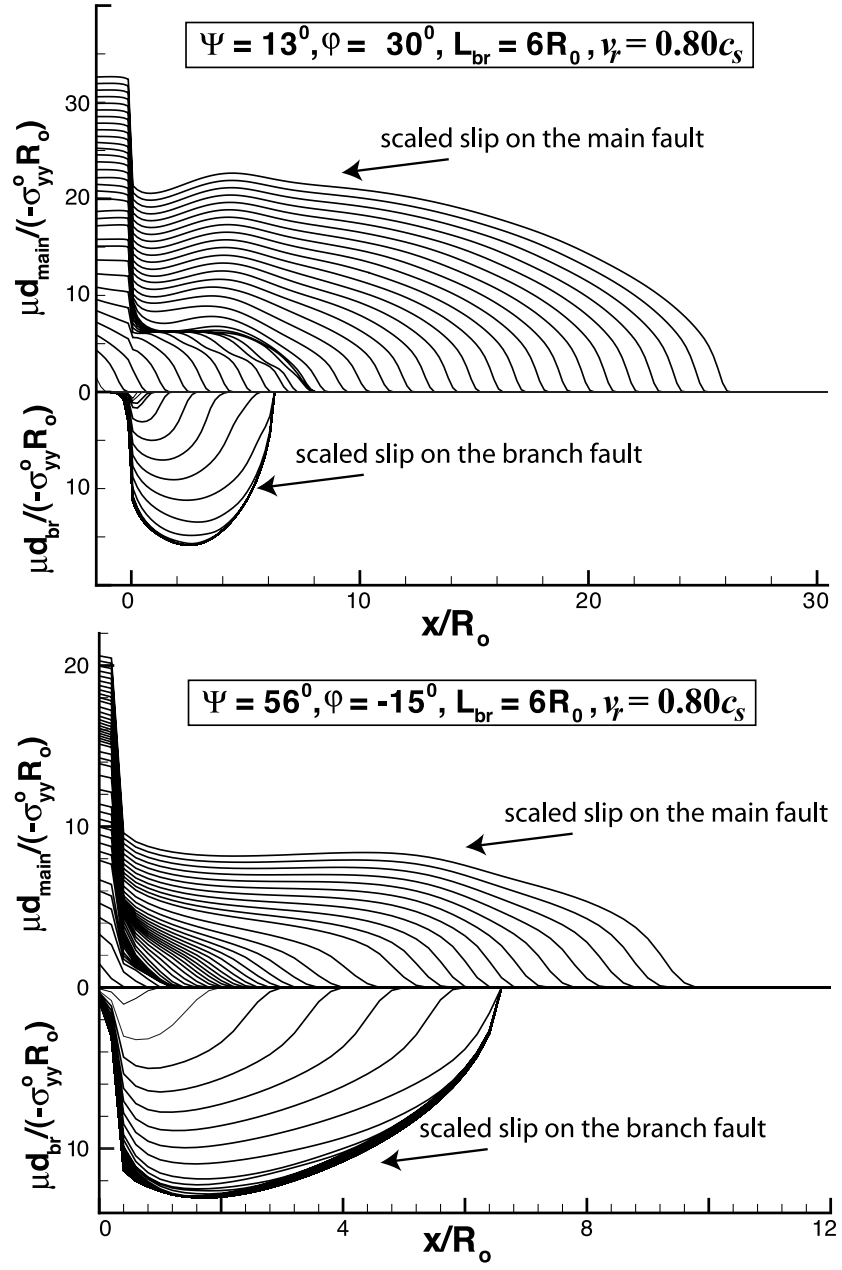

Figure 15. Complexities in normalized slip, around the branching junction, for both the main and the branch fault. Here $d$ is the slip, $\mu, \sigma_{y y}^{o}, L_{b r}$ are the shear modulus, initial fault normal stress and the length of the branch respectively.

compressive stress, $\Psi$, approached its extrema, $\Psi=0^{\circ}$ or $90^{\circ}$, even the high rupture velocity cases led to exclusive branching.

[67] In this body of work we considered the role of the length of the branches on rupture propagation characteristics on the main fault. We studied the cases by Kame et al. [2003] and Bhat et al. [2004] where branching was observed and terminated rupture on the branched fault at various distances from the branching junction. A dynamically propagating rupture when stopped radiates stress perturbations as it tries to establish a static stress field. The zone of influence of this static-like field depends on many parameters including the length of the rupture. Harris and Day [1993], Harris et al. [2002] and Fliss et al. [2005] have studied the phenomenon of rupture jumping to an adjacent fault due to stoppage on the main fault. In our studies, with finite branched systems, we thus expect some complexity in the rupture propagation process due to the stoppage of rupture on the branch.

[68] We have noticed that for branches on the compressional side termination of rupture on the same barely affects the rupture on the main fault except for the case when $\Psi=13^{\circ}, v_{r}=0.80 c_{s}, \varphi=30^{\circ}$ and $L_{b r}=30 R_{0}$. For the above case we pointed out that a propitious combination of parameters led to the direct interaction between the rupture front on the main fault and the large stress perturbation from the branch end led to the termination of rupture on the main fault. A short or an infinite branch would have allowed for the rupture to continue on the main fault [Figure 12].

[69] For branches on the extensional side, while an infinite branch would have allowed continuation of rupture on the main fault for high incoming rupture velocity and shallow branch angle, termination of rupture on the branch led to its continuation on the main fault for and $\Psi=70^{\circ}$, $L_{b r}=6,20 R_{0}$. We also showed that, for the above cases, when $v_{r}$ was reduced to $0.80 c_{s}$ terminating the rupture on the branch closer to the main fault led to re-nucleation of rupture on the main fault.

[70] We observed complexities in slip distribution and rupture velocity evolution on the main fault. The complexities in the slip distribution process, which might normally have been attributed to a rupture encountering a barrier or an asperity were the result of rupture propagation and termination on the main fault. Complexities in rupture
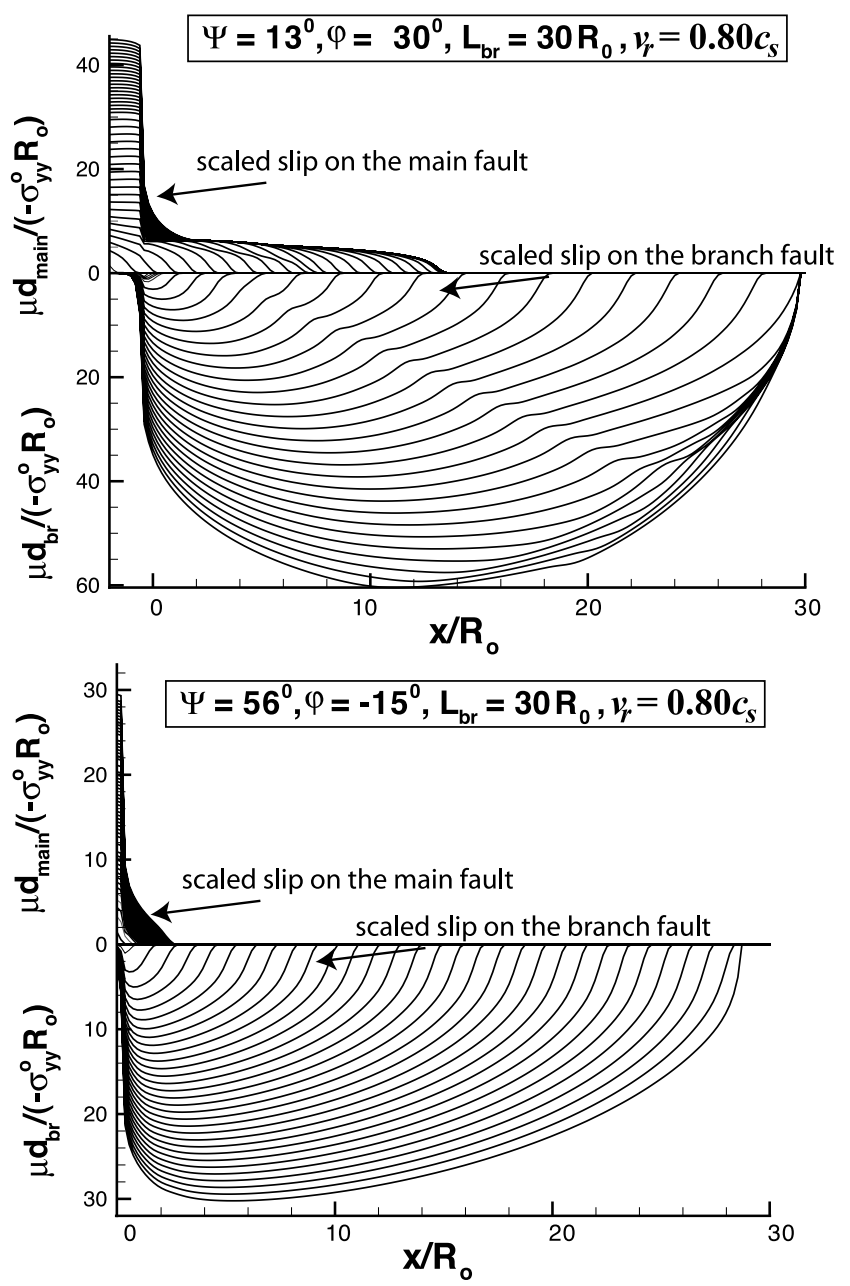

Figure 16. Complexities in normalized slip, around the branching junction, for both the main and the branch fault. Here $d$ is the slip, $\mu, \sigma_{y y}^{o}, L_{b r}$ are the shear modulus, initial fault normal stress and the length of the branch respectively. 

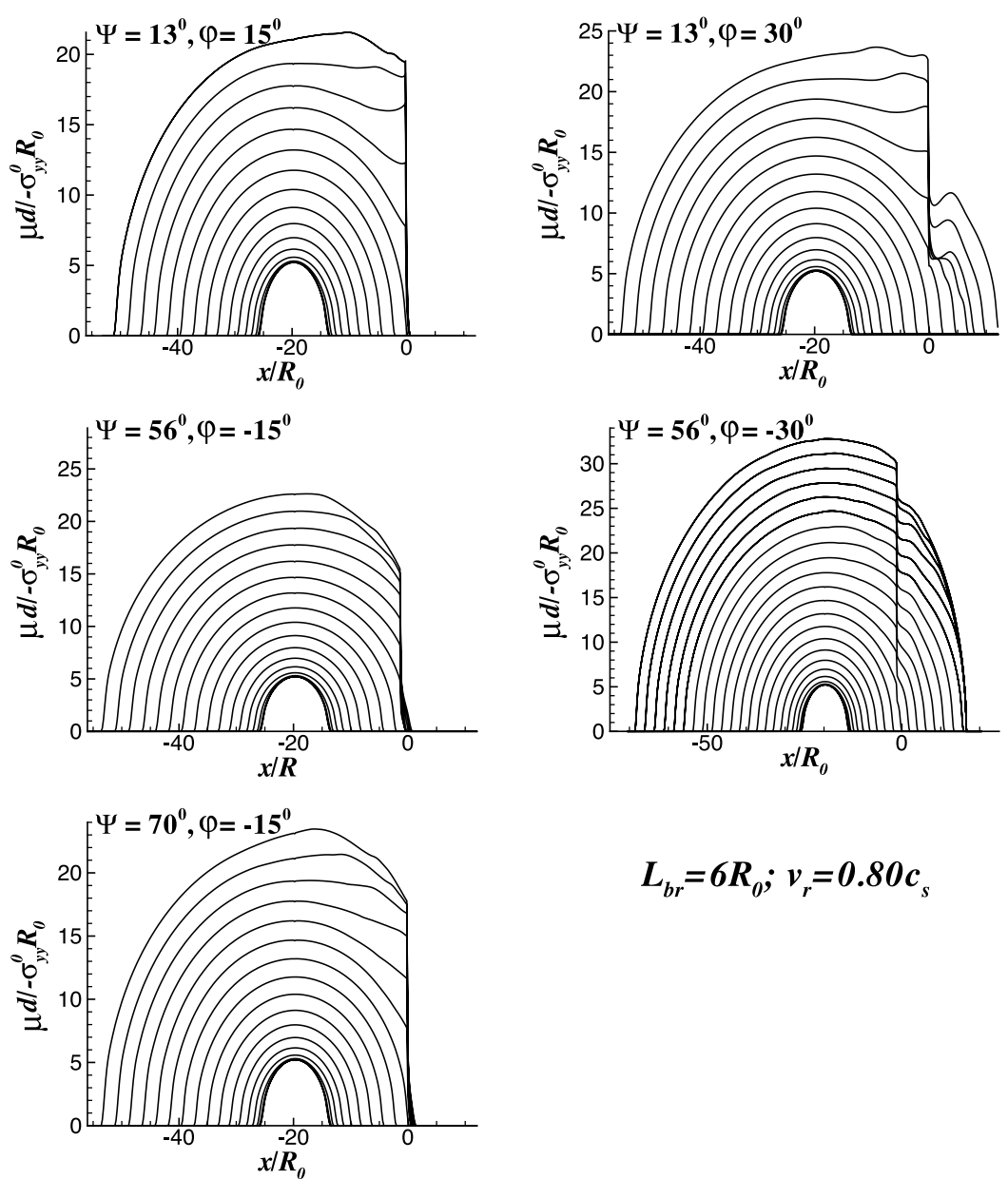

$$
L_{b r}=6 R_{0} ; v_{r}=0.80 c_{s}
$$

Figure 17. Complexities in normalized slip on the main fault when the rupture has terminated on the branch and a static-like stress field is established around it. Here $d$ is the slip, $\mu, \sigma_{y y}{ }^{o}, L_{b r}$ are the shear modulus, initial fault normal stress and the length of the branch respectively.

velocity evolution, like deceleration and acceleration of rupture, are directly associated with the finiteness of the branch and could be the source of the high-frequency content of strong ground motion.

\section{Appendix: Evaluating Rupture Velocity Evolution in Numerical Dynamic Rupture Propagation Procedures}

[71] Due to the discretized (spatial and temporal) nature of the numerical procedure the rupture tip progress is quantized. In other words the incremental rupture tip advance is through a distance equal to the length of the spatial cell over a variable, but necessarily integer, number of time steps. This leads to a staircase like data-set [(Figure A1a)] for rupture tip advance over time. Direct numerical differentiation of this data to obtain rupture velocity evolution with time is hence not recommended.

[72] In earlier work Bhat et al. [2004] the above mentioned issue with numerical differentiation was partially overcome by evaluating the rupture velocity in the following way. They calculated the average time $\left(\Delta t_{\text {avg }}\right)$ taken for the rupture tip to advance by three spatial cells $(3 \Delta s)$. The rupture velocity, $v_{r}$, is then simply given by $v_{r}=3 \Delta s / \Delta t_{\text {avg }}$.
The procedure is robust enough for calculations where there are no abrupt changes in the crack-tip advance history and produces a relatively good rupture velocity evolution data. In the current work we noticed several abrupt transitions in the rupture tip history data and the above mentioned algorithm would fail to capture such transitions.

[73] We instead use locally weighted quadratic polynomial regression [Cleveland, 1979] to smooth the rupture tip position versus time data [(Figure A1b)]. At each point in the data set a quadratic polynomial is fit to a subset of the data using weighted least squares method, giving more weight to points near the point whose response is being estimated and less weight to points further away. The value of the regression function for the point is then obtained by evaluating the local polynomial. The resulting data-set was then numerically differentiated using a forward difference scheme to calculate the rupture velocity evolution data [(Figure A1c)]. This data were further smoothed using the locally weighted quadratic polynomial regression method to produce the rupture velocity evolution plot [(Figure A1d)].

[74] We also used the above algorithm to verify the results obtained by Bhat et al. [2004], Figure 12. While the resulting rupture velocity curve was significantly 
(a)

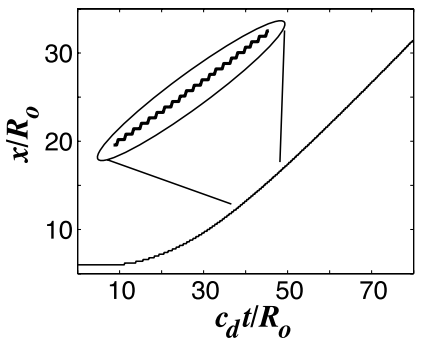

(c)

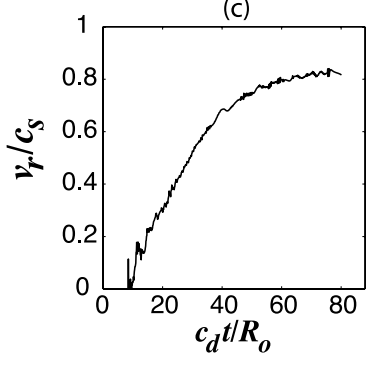

(b)

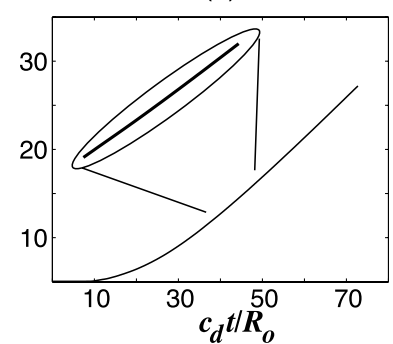

(d)

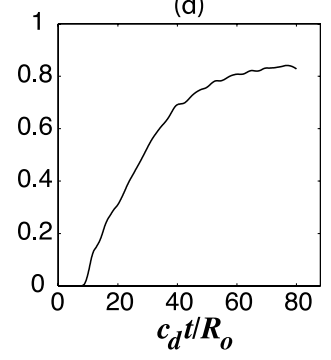

Figure A1. Data smoothing for rupture tip position and rupture velocity data. (a) Rupture tip position versus time before smoothing (Oval inset shows zoomed in plot). (b) Rupture tip position versus time after locally weighted polynomial regression smoothing (Oval inset shows zoomed in plot). (c) Rupture velocity evolution obtained by numerically differentiating A1(b). (d) Rupture velocity after locally weighted polynomial regression smoothing.

smoother the general conclusions obtained by Bhat et al. [2004] agrees with the smoothed data.

[75] Acknowledgments. We are very grateful for helpful reviews and comments from Gilbert Brietzke and Steven Day. We thank Charles G. Sammis for questioning during an informal presentation which led to our improved procedures of the Appendix for rupture velocity versus time plots. This study was supported at Harvard University by NSF-EAR award 0105344 and by the Southern California Earthquake Center (SCEC), funded by NSF Cooperative Agreement EAR-0106924 and USGS Cooperative Agreement 02HQAG0008 (this is SCEC Contribution 1066).

\section{References}

Andrews, D. J. (1976), Rupture velocity of plane strain shear cracks, J. Geophys. Res., 81, 5679-5687.

Aochi, H., and E. Fukuyama (2002), Three-dimensional non-planar simulation of the 1992 Landers earthquake, J. Geophys. Res., 107(B2), 2035, doi:10.1029/2000JB000061.

Aochi, H., E. Fukuyama, and M. Matsuura (2000a), Spontaneous rupture propagation on a non-planar fault in 3D elastic medium, Pure Appl. Geophys., 157, 2003-2027.

Aochi, H., E. Fukuyama, and M. Matsuura (2000b), Selectivity of spontaneous rupture propagation on a branched fault, Geophys. Res. Lett., 27, $3635-3638$.

Aochi, H., R. Madariaga, and E. Fukuyama (2002), Effect of normal stress during rupture propagation along nonplanar faults, J. Geophys. Res., 107(B2), 2038, doi:10.1029/2001JB000500.

Aochi, H., O. Scotti, and C. Berge-Thierry (2005), Dynamic transfer of rupture across differently oriented segments in a complex 3-D fault system, Geophys. Res. Lett., 32, L21304, doi:10.1029/2005GL024158.

Aydin, A., and R. A. Schultz (1990), Effect of mechanical interaction on the development of strike-slip faults with echelon patterns, J. Struct. Geol., $12,123-129$.

Bhat, H. S., R. Dmowska, J. R. Rice, and N. Kame (2004), Dynamic slip transfer from the Denali to Totschunda faults, Alaska: Testing theory for fault branching, Bull. Seismol. Soc. Am., 94(6B), S202-S213.

Bhat, H. S., R. Dmowska, G. C. P. King, Y. Klinger, and J. R. Rice (2007), Off-fault damage patterns due to supershear ruptures with application to the $2001 \mathrm{Mw} 8.1$ Kokoxili (Kunlun) Tibet earthquake, J. Geophys. Res., 112, B06301, doi:10.1029/2006JB004425.

Brankman, C. M., and A. Aydin (2004), Uplift and contractional deformation along a segmented strike-slip fault system: the Gargano Promontory, southern Italy, J. Struct. Geol., 26, 807-824.

Cleveland, W. S. (1979), Robust locally weighted regression and smoothing scatterplots, J. Am. Stat. Assoc., 74, 829-836.

Cochard, A., and R. Madariaga (1994), Dynamic faulting under ratedependent friction, Pure Appl. Geophys., 142, 419-445.

Dreger, D. S., D. D. Oglesby, R. Harris, N. Ratchkovski, and R. Hansen (2004), Kinematic and dynamic rupture models of the November 3, 2002 Mw7.9 Denali, Alaska, earthquake, Geophys. Res. Lett., 31, L04605, doi:10.1029/2003GL018333.

Duan, B., and D. D. Oglesby (2005), Multicycle dynamics of nonplanar strike-slip faults, J. Geophys. Res., 110, B03304, doi:10.1029/ 2004JB003298

Fliss, S., H. S. Bhat, R. Dmowska, and J. R. Rice (2005), Fault branching and rupture directivity, J. Geophys. Res., 110, B06312, doi:10.1029/ 2004JB003368.

Harris, R. A., and S. M. Day (1993), Dynamics of fault interaction: Parallel strike-slip faults, J. Geophys. Res., 98, 4461-4472.

Harris, R. A., and S. M. Day (1999), Dynamic 3D simulations of earthquakes on en echelon faults, Geophys. Res. Lett., 26, 2089-2092.

Harris, R. A., R. J. Archuleta, and S. M. Day (1991), Fault steps and the dynamic rupture process: 2 -d numerical simulations of a spontaneously propagating shear fracture, Geophys. Res. Lett., 18, 893-896.

Harris, R. A., J. F. Dolan, R. Hartleb, and S. M. Day (2002), The 1999 Izmit, Turkey, earthquake: A 3D dynamic stress transfer model of intraearthquake triggering, Bull. Seismol. Soc. Am., 92, 245-255.

Heaton, T. H. (1990), Evidence for and implications of self-healing pulses of slip in earthquake rupture, Earth Planet. Int., 64, 1-20.

Ida, Y. (1972), Cohesive force across the tip of a longitudinal-shear crack and Griffith's specific surface energy, J. Geophys. Res., 77, 3796-3805.

Kame, N., and T. Yamashita (1999a), Simulation of the spontaneous growth of a dynamic crack without constraints on the crack tip path, Geophys. J. Int., 139, 345-358.

Kame, N., and T. Yamashita (1999b), Simulation of spontaneous growth of dynamic crack without constraints on the crack tip path, Geophys. J. Int., 139, 349-358.

Kame, N., J. R. Rice, and R. Dmowska (2003), Effects of pre-stress state and rupture velocity on dynamic fault branching, J. Geophys. Res., 108(B5), 2265, doi:10.1029/2002JB002189, pp. ESE 13-1 to 13-21. Download: http://esag.harvard.edu/dmowska/KRD.pdf

King, G. C. P. (1986), Speculations on the geometry of the initiation and termination processes of earthquake rupture and its relation to morphology and geological structure, Pure Appl. Geophys., 124, $567-585$.

King, G., and J. Nabelek (1985), Role of fault bends in the initiation and termination of earthquake rupture, Science, $228,984-987$.

Knuepfer, P. L. K. (1989), Implications of the characteristics of end-points of historical surface fault ruptures for the nature of fault segmentation, U.S. Geol. Surv. Open File Rep., 89-315, 193-228.

Koller, M., M. Bonnet, and R. Madariaga (1992), Modelling of dynamical crack propagation using regularized time-domain boundary integral equation, Wave Motion, 16, 339-366.

Madariaga, R. (1977), High-frequency radiation from cracks (stress-drop) models of earthquake faulting, Geophys. J. R. Astron. Soc., 51, 625-651.

Oglesby, D. D. (2005), The dynamics of strike-slip stepovers with linking dip-slip faults, Bull. Seismol. Soc. Am., 95(5), 1604-1622, doi:10.1785/ 0120050058

Oglesby, D. D., S. M. Day, Y.-G. Li, and J. E. Vidale (2003), The 1999 Hector Mine earthquake: The dynamics of a branched fault system, Bull. Seismol. Soc. Am., 93(6), 2459-2476, doi:10.1785/0120030026.

Olsen, K. B., R. Madariaga, and R. J. Archuleta (1997), Three-dimensional dynamic simulation of the 1992 Landers earthquake, Science, 278(5339), 834-837.

Palmer, A. C., and J. R. Rice (1973), The growth of slip surfaces in the progressive failure of over-consolidated clay, Proc. R. Soc. London, A332, 527-548.

Poliakov, A. N. B., R. Dmowska, and J. R. Rice (2002), Dynamic shear rupture interactions with fault bends and off-axis secondary faulting, J. Geophys. Res., 107(B11), 2295, doi:10.1029/ 2001JB000572, pp. ESE 6-1 to 6-18.

Rice, J. R. (1968), Mathematical analysis in the mechanics of fracture, in Fracture: An Advanced Treatise (Vol. 2, Mathematical Fundamentals), Chapter 3, edited by H. Liebowitz, pp. 191-311, Academic Press, New York.

Rice, J. R. (1980), The mechanics of earthquake rupture, in Physics of the Earths Interior (Proc. International School of Physics 'Enrico Fermi', Course 78, 1979), edited by A. M. Dziewonski and E. Boschi, pp. 555-649, Elsevier, Italian Physical Society and North-Holland Publ. Co., New York. 
Rice, J. R. (1993), Spatio-temporal complexity of slip on a fault, J. Geophys. Res., 98, 9885-9907.

Rice, J. R., C. G. Sammis, and R. Parsons (2005), Off-fault secondary failure induced by a dynamic slip-pulse, Bull. Seismol. Soc. Am., 95(1), 109134, doi:10.1785/0120030166.

Sibson, R. H. (1985), Stopping of earthquake ruptures at dilational fault jogs, Nature, 316, 248-251.

Sibson, R. H. (1986), Rupture interactions with fault jogs, in Earthquake Source Mechanics, Geophys. Monogr. Ser., vol. 37, edited by S. Das, J. Boatwright, and C. H. Scholz, pp. 157-167, AGU, Washington, D. C.

Sowers, J. M., J. R. Unruh, W. R. Lettis, and T. D. Rubin (1994), Relationship of the Kickapoo fault to the Johnson Valley and Homestead Valley Faults, San Bernardino County, California, Bull. Seismol. Soc. Am., 84 $528-536$.

Tada, T., and T. Yamashita (1997), Non-hypersingular boundary integral equations for two-dimensional non-planar crack analysis, Geophys. J. Int., 130(2), 269-282.

Wesnousky, S. G. (1988), Seismological and structural evolution of strikeslip faults, Nature, 335, 340-343.
Wesnousky, S. G. (2006), Predicting the endpoints of earthquake ruptures, Nature, 144, doi:10.1038/nature05275.

Yamashita, T., and Y. Umeda (1994), Earthquake rupture complexity due to dynamic nucleation and interaction of subsidiary faults, Pure Appl. Geophys., 143, 89-116.

Yule, D., and K. E. Sieh (2003), Complexities of the San Andreas fault near San Gorgonio Pass: Implications for large earthquakes, J. Geophys. Res., 108(B11), 2548, doi:10.1029/2001JB000451.

H. S. Bhat, School of Engineering and Applied Sciences, Harvard University, 327 Pierce Hall, 29 Oxford Street, Cambridge, MA 02138, USA. (bhat@esag.harvard.edu)

R. Dmowska and J. R. Rice, School of Engineering and Applied Sciences, Harvard University, 227 Pierce Hall, 29 Oxford Street, Cambridge, MA 02138, USA. (dmowska@esag.harvard.edu; rice@esag.harvard.edu)

M. Olives, Laboratoire de Mcanique, Ecole Polytechnique, 91128 Palaiseau, France. (molives@esag.harvard.edu) 